\title{
PARASOL in-flight calibration and performance
}

\author{
Bertrand Fougnie, ${ }^{1, \star}$ Guillaume Bracco, ${ }^{2}$ Bruno Lafrance, ${ }^{3}$ Caroline Ruffel, ${ }^{3}$ Olivier Hagolle, ${ }^{1}$ \\ and Claire Tinel $^{1}$ \\ ${ }^{1}$ Centre National d'Etudes Spatiales, 18 avenue E. Belin, 31401 Cedex 9, Toulouse, France
2Magellium, 24 rue Hermes, 31520 Ramonville Saint-Agne, France
${ }^{3}$ CS-Systèmes d'Information, rue Brindejonc des Moulinais, 31506 Cedex 5, Toulouse, France \\ ${ }^{\star}$ Corresponding author: Bertrand.Fougnie@cnes.fr
}

Received 7 February 2007; revised 30 May 2007; accepted 31 May 2007;

posted 1 June 2007 (Doc. ID 79784); published 23 July 2007

\begin{abstract}
Since 18 December 2004, the PARASOL satellite is a member of the so-called A-train atmospheric orbital observatory, flying together with Aqua, Aura, CALIPSO, CLOUDSAT, and OCO satellites. These satellites combine for the first time a full suite of instruments for observing aerosols and clouds, using passive radiometer complementarily with active lidar and radar sounders. The PARASOL payload is extensively derived from the instrument developed for the POLDER programs that performs measurements of bidirectionality and polarization for a very wide field-of-view and for a visible/near-infrared spectral range. An overview of the results obtained during the commissioning phase and the reevaluation after one year in orbit is presented. In-flight calibration methods are briefly described, and radiometric and geometric performances are both evaluated. All algorithms are based on a panel of methods using mainly natural targets previously developed for POLDER missions and adapted or redeveloped in the PARASOL context. Regarding performances, all mission requirements are met except for band 443 (not recommended for use). After one year in orbit, a perfect geometrical stability was found while a slight decrease of the radiometric sensitivity was observed and corrected through an innovative multitemporal algorithm based on observations of bright and scattered convective clouds. The scientific exploitation of PARASOL has now begun, particularly by coupling these specific observations with other A-train sensor measurements. (C) 2007 Optical Society of America

OCIS codes: $\quad 010.1290,010.1310,120.0120,120.5410,280.1310$.
\end{abstract}

\section{Introduction}

In 1999, the unique scientific opportunity to add into the so-called A-train a POLDER designed instrument [1] onboard a CNES Myriade microsatellite [2] was identified. Thanks to its unique capability to perform multiangular and polarized acquisitions combined with the spectral information, PARASOL (Polarization \& Anisotropy of Reflectance for Atmospheric Sciences coupled with Observations from a Lidar) is able to characterize the radiative properties of clouds and aerosols. In addition, the complementarity with other sensors flying in the A-train formation opens a large window of possibilities combining the various sets of acquisitions from CERES and MODIS radiometers, the lidar on CALIPSO, and the radar on CLOUDSAT.

0003-6935/07/220001-17\$15.00/0

(C) 2007 Optical Society of America
This "afternoon constellation" provides a unique atmospheric observatory for aerosol and clouds characterization fundamental for a better knowledge of their radiative impact and to improve our understanding of climate and climate change.

Table 1, the PARASOL payload, is a heritage of POLDER instruments [1] carried onboard ADEOS 1 and 2 in 1996-1997 and 2002-2003. This innovative concept is a combination of polarization and multidirectional acquisitions: a complete description of the polarization is available for 3 spectral bands and for up to 16 viewing angles. As shown in Table 2, the spectral signature of the target is assessed for 9 spectral bands in the $443-1020 \mathrm{~nm}$ spectral range. For each of the polarized bands, 490, 670, or 865 , three acquisitions are performed through a polarizer oriented with $-60^{\circ}, 0^{\circ}$, and $60^{\circ}$ respectively from a given reference, from which are retrieved the Stokes parameters $[I, Q, U]$. The major modification made on 
Table 1. Main Characteristics for PARASOL Satellite and Payload

\begin{tabular}{ll} 
Launch date & 18th December 2004 \\
Platform & Myriad \\
Altitude & $705 \mathrm{~km}$ \\
Local time & $13 \mathrm{~h} 30$ \\
Mass & $120 \mathrm{~kg}$ \\
Size & $0.6 \times 0.8 \times 0.8 \mathrm{~m}$ \\
Instrument & POLDER \\
Spectral bands & 9 \\
Polarized bands & 3 \\
Spectral range & $443-1020 \mathrm{~nm}$ \\
Detector & CCD Matrix $242 \times 274$ \\
Swath & $1600 \mathrm{~km}$ cross-track \\
& $2100 \mathrm{~km}$ along-track \\
Resolution & $6.18 \mathrm{~km}$ (level-1 grid) \\
Field-of-view & $\pm 57^{\circ}$ \\
\hline
\end{tabular}

the instrument compared to POLDER 1 and 2 was a $90^{\circ}$ rotation of the CCD matrix allowing PARASOL to perform more acquisitions along-track to enhance the characterization of the bidirectionality of targets. As a result, the swath is reduced to $1600 \mathrm{~km}$ cross track (Table 1) compared to $2200 \mathrm{~km}$ for POLDER 1 and 2.

PARASOL was launched 18 December 2004, and the first image was acquired 7 January 2005. The commissioning phase started with 2 months of specific programming of both payload and satellite for calibration and image quality purposes and mission standard acquisitions started on 4 March 2005. The commissioning phase ended 12 July 2005 with the image quality review. After one year of operational use, a general review was made confirming the excellent health and performances of the PARASOL system [3]. A reprocessing of the level-1 archive, which included a correction of a light temporal decrease of the radiometric sensitivity, was performed the end of 2006 .

The goal of this paper is an overview of results from calibration algorithms, characterization methods, and performances for both geometric and radiometric aspects. Because most of methods were previously described in other papers, mainly in Hagolle et al. [4] for radiometric aspects applied to the POLDER/ ADEOS-1 sensor, the present paper does not detail each algorithm but only provides brief descriptions and focuses on particularity or innovative adaptations for PARASOL.

\section{Radiometric Calibration and Performance}

\section{A. Preflight Characterization}

For a given spectral band $k$, the radiometric model (simplified version including polarization) describes the physical response of the instrument to the incoming signal characterized by $[I, Q, U]$ in the Stokes's formalism [5]. It can be written

$$
\begin{aligned}
X_{l, p}= & C_{l, p}+A_{k} g_{l, p} G t_{i} P_{l, p}\left[p_{1}\left(l, p, \varepsilon, \eta, \alpha^{a}, T^{a}\right) I\right. \\
& +p_{2}\left(l, p, \varepsilon, \eta, \alpha^{a}, T^{a}\right) Q \\
& \left.+p_{3}\left(l, p, \varepsilon, \eta, \alpha^{a}, T^{a}\right) U\right],
\end{aligned}
$$

where

- $l, p$ are the pixel coordinates,

- $X_{l, p}$ is the numerical count measured for pixel $(l, p)$,

- $C_{l, p}$ is the dark current for pixel $(l, p)$,

- $[I, Q, U]$ are Stokes parameters expressed on a given reference axis,

- $A_{k}$ is the absolute calibration coefficient,

- $G$ is the electronic gain,

- $t_{i}$ is the integration time,

- $P_{l, p}$ is the low-frequency polynomial,

- $g_{l, p}$ is the high-frequency interpixel coefficient,

and where $p_{1}, p_{2}$, and $p_{3}$ are depending on pixel coordinates, $l$ and $p$, the optic sensitivity to polarization, $\varepsilon$, and for polarized channels $a$ the polarizer extinction ratio, $\eta$, orientation, $\alpha^{a}$, and transmission, $T^{a}$. This radiometric model, its inversion to retrieve $[I, Q, U]$, and the full level-1 data processing are precisely described in Hagolle et al. [5]. The preflight radiometric calibration of the instrument consists in accurate estimation of each of these parameters. Procedures and ground equipments are described in Ref. 6 , and some processing algorithms were improved and updated in Ref. 7. Parameters are usually classified into the following families:

- Absolute calibration is the estimation of $A_{k}$, a global parameter independent of the considered pixel.

- In-polarization calibration refers to estimation of optic and polarizer sensitivity, i.e., estimation of $T^{a}$, $\varepsilon, \eta$, and $\alpha^{a}$ parameters.

- Multiangular calibration refers for each spectral band to variation of the calibration with viewing angle

\begin{tabular}{|c|c|c|c|c|c|c|c|c|c|}
\hline & \multicolumn{9}{|c|}{ Spectral Band } \\
\hline & 443 & 490 & 565 & 670 & 763 & 765 & 865 & 910 & 1020 \\
\hline Central wavelength (nm) & 443.5 & 490.9 & 563.8 & 669.9 & 762.9 & 762.7 & 863.7 & 907.1 & 1019.6 \\
\hline Bandwidth (nm) & 13.4 & 16.3 & 15.4 & 15.1 & 10.9 & 38.1 & 33.7 & 21.1 & 17.1 \\
\hline Polarization & - & yes & - & yes & - & - & yes & - & - \\
\hline Saturation level (reflectance) & 1.28 & 0.99 & 1.06 & 1.01 & 1.04 & 0.96 & 1.00 & 0.98 & 1.70 \\
\hline
\end{tabular}

Table 2. PARASOL Spectral Bands Including Central Wavelength, Bandwidth, Ability to Measure Polarization, and Saturation Level in Reflectance Unit 
or pixel, i.e., estimation of $g_{l, p}$ and $P_{l, p}$ (when speaking about equalization, $C_{l, p}$ is also considered). Qualitatively, the polynomial $P_{l, p}$ refers to low-frequency variations of the optic transmission slightly decreasing when the viewing angle is increasing, while the coefficient $g_{l, p}$ mainly refers to high-frequency variations of the sensitivity of elementary detectors or to variations of the optic transmission that cannot be modeled as a polynomial function. These aspects are detailed for wide field-of-view sensors similar to PARASOL in Ref. 4 (POLDER) and Ref. 8 (Végétation).

Figure 1 illustrates typical values for $P_{l, p}($ or $P(\theta))$ and $\varepsilon(\theta): P_{l, p}$ varies from 1 for nadir viewing to 0.88 at large viewing angles of $55^{\circ}$, i.e., a $12 \%$ decrease of the sensitivity, while $\varepsilon(\theta)$ varies from 0 at nadir to about $4 \%$ at $55^{\circ}$. Multiangular $g_{l, p}$ coefficients vary from 0.98 to 1.02 over the matrix for which the mean value is 1.0 .

A full characterization of the spectral response of the instrument (hereafter called SRI) is required in order to guarantee the efficiency of in-flight calibration methods, but also to guarantee an optimized scientific analysis over Earth-atmosphere targets behaving strongly differing spectral signatures as shown in Fig. 2. As no in-flight adjustment of the SRI will be possible, various aspects of the SRI were analyzed with appropriate preflight sets of measurement: variability of the SRI into the field-of-view (typically $0.2 \mathrm{~nm}$ ), spectral rejection (about $0.2 \%$ ), variability over a given polarized triplet (better than $\pm 0.2 \mathrm{~nm}$ ), and expected variation in the space vacuum environment (less than $0.2 \mathrm{~nm}$ ). The mean SRIs derived from the preflight campaign are given in Table 2.

As described in Fougnie et al. [9], a light nonlinearity of the detector response was identified, mod-

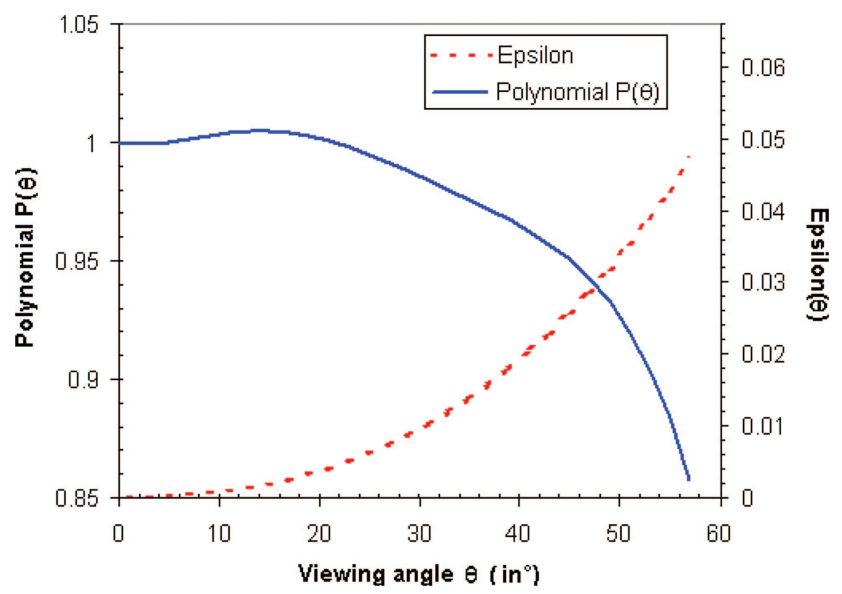

Fig. 1. (Color online) Typical shapes and values for the polynomial $P(\theta)$ or $P_{l, p}$ and epsilon $\varepsilon(\theta)$ as a function of the viewing angle $\theta$. Polynomial $P(\theta)$ is linked to $P_{l, p}$ through $\tan (\theta)=(1 / f) \operatorname{sqrt}[(l$ $-137.5) \mathrm{d} l+(p-121.5) \mathrm{d} p]$ with $f=3.55 \mathrm{~mm}$ is the focal distance of the instrument, $\mathrm{d} l=32$ and $\mathrm{d} p=27$ micrometers are sizes of a CCD pixel, $l$ and $p$ are CCD line and column numbers. For the nadir direction, $P(\theta)$ is normalized to 1 and $\varepsilon(\theta)$ is null.

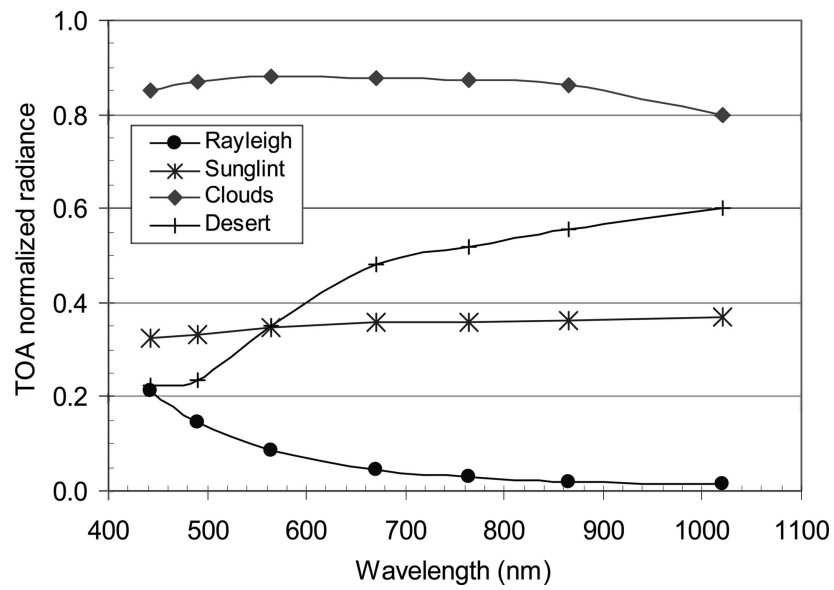

Fig. 2. Typical top-of-atmosphere normalized radiances for the PARASOL spectral bands and corresponding to targets selected for calibration over Rayleigh scattering, sunglint, clouds, and desert sites. TOA normalized radiances, presented after gaseous absorption correction for ozone, oxygen, and water vapor, illustrate differences between the various calibration targets for both spectral behavior and radiance level aspects.

eled as a function and corrected in the level-1 processing. The preflight characterization of this function is done using several acquisitions of the same source for a great number of integration times. This nonlinearity function is illustrated Fig. 9 showing that neglect this correction would lead to $\pm 1 \%$ error on the retrieved normalized radiance $I$.

PARASOL is an instrument composed of a single wide field-of-view camera [1]. Such instrumental concepts are usually concerned by stray-light phenomena. A stray-light correction, described in Ref. 5 or Ref. 10 and included on the PARASOL level-1 processing algorithm, requires a dedicated characterization though a heavy preflight calibration campaign. A serious problem appeared for the band 443 for which it is supposed that a default occurred specifically for this spectral band on the antireflect coating of one of the several diopters composing the filterwheel. Consequently, the stray-light intensity was found to be 5 to 10 times greater than other spectral bands and the correction algorithm on level-1 processing failed: unfortunately, the band 443 is with poor accuracy. It is recommended to PARASOL data users not to use this spectral band and we will discuss no more the case of the band 443 in the following of this paper.

\section{B. Absolute Calibration}

\section{Description of Methods}

The POLDER instrument has no on-board calibration device to transfer-to-orbit the preflight calibration. But nevertheless, even if the space sensor is able to deliver an autonomous on-board calibration, it has to be validated and controlled through vicarious methods; see examples in Eplee et al. [11] for SeaWiFs, Hagolle et al. [12] for MERIS, or Fougnie et al. [13] and Hagolle et al. [4] for POLDER-1. Consequently, in- 
tensive efforts have historically been made to develop calibration methods based on acquisitions over natural targets. Four particular natural targets from the Earth-atmosphere system were privileged for their specific characteristics. In such methods, the approach is first to accurately compute the top-ofatmosphere (TOA) reference signal that the sensor of interest should observe over a selected target and secondly, to compare the reference signal to the signal really observed and delivered by the satellite sensor. These methods are:

(a) Absolute calibration over Rayleigh scattering: The TOA signal measured over oceanic sites, i.e., dark surface, is due at $90 \%$ to scattering by atmospheric molecules, and this is the reason why the spectral behavior of the TOA signal is strongly varying with the wavelength $\lambda$, very closely to a $\lambda^{-4}$ law (see Fig. 2). In order to accurately compute this dominant Rayleigh scattering contribution, the equivalent Rayleigh optical thickness has to be calculated for each spectral band using surface pressure and the SRI (see, e.g., Ref. 13). Nevertheless, an absolute calibration can be derived only if other contributors to the TOA signal are controlled: clouds, aerosols, gaseous absorption, surface phenomena, and marine reflectances. Oligotrophic oceanic sites, illustrated on Fig. 3, were selected for their homogeneities and moderated seasonal variations, and were characterized through a climatology of marine reflectance [14]. Cloud and aerosol masks are based on a strict threshold using the band 865. A correction of the residual aerosol content is made assuming a Maritime-98\% aerosol model from Shettle and Fenn [15] and using the background aerosol optical thickness measured by the band 865 with a typical mean value of 0.025 . This method used to calibrate spectral bands up to
$670 \mathrm{~nm}$ is originally derived from Vermote et al. [16] and is described in Hagolle et al. [4].

(b) Interband calibration over sunglint: The reflexion of the sun over the oceanic surface observed from space is a bright and nearly spectrally white phenomenon as illustrated in Fig. 2. An absolute calibration over sunglint should require an accurate modeling of the sunglint radiance which is strongly dependent on the sea surface roughness (as described by Cox and Munk [17] and in practice too difficult to assess. In fact, the white spectral behavior shown in Fig. 2 can be used to provide an interband calibration by simply comparing the signal measured in various spectral bands to the signal measured in a band used as reference. Some corrections are nevertheless necessary: contribution and transmission of molecular scattering, gaseous absorption, marine reflectance (same oceanic sites defined Fig. 3 for Rayleigh scattering), and aerosol background. The multidirectional capability of PARASOL is used to efficiently discard measurements perturbed by aerosols using a threshold on out-of-glint viewing direction. The reference band to use should be well calibrated, and a red band (here $670 \mathrm{~nm}$ ) is usually selected because of lower biases into the absolute calibration previously derived from Rayleigh scattering method and lower atmospheric contribution than for shorter wavelengths. Another argument is that the intercalibration error increases with spectral distance from the reference band, and the red band is at the middle of the spectral range of the instrument (see Table 2). Spectral bands from 490 to 1020 can be intercalibrated with the band 670. Principle of the method is described and applied to POLDER-1 in Hagolle et al. [4], updated in Hagolle et al. [18] in the case of VEGETATION, and ap-

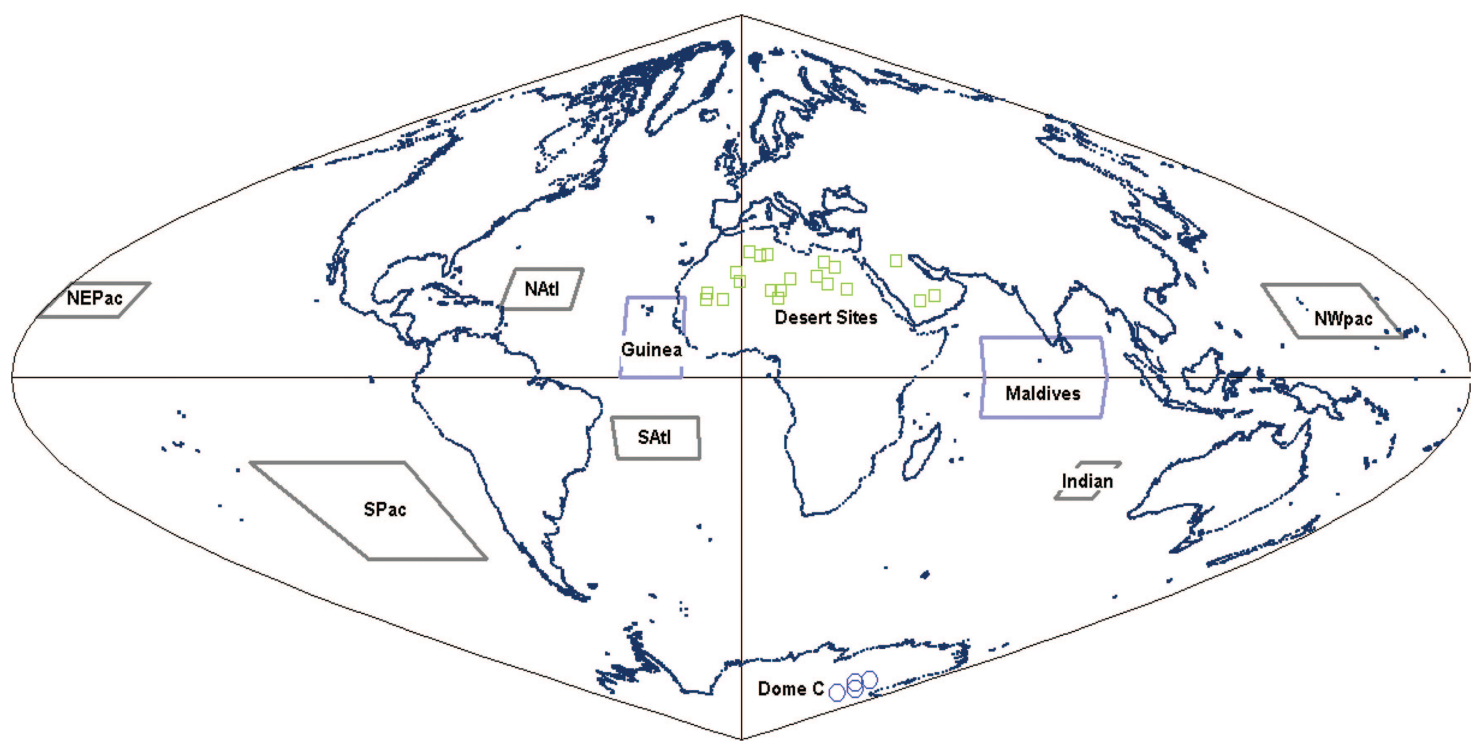

Fig. 3. (Color online) Location of calibration sites: oceanic sites used for Rayleigh scattering and sunglint calibrations (Indian, North/ South Pacific, and Atlantic), oceanic sites used for calibration over bright clouds (Maldives and Guinea), desert sites used for cross calibration with other sensors (20 sites in Africa and Arabia), and Antarctic sites used for multitemporal calibration (4 sites in Dome C, not presented on this paper). 
plied for calibration of POLDER polarized channel in Toubbé et al. [19].

(c) Interband calibration over clouds: High and dense convective clouds are strongly reflecting the downwelling irradiance of the sun, and such clouds can be assumed white and Lambertian with a very good approximation as illustrated in Fig. 2. Using a spectral band as reference to characterize the reflectance of the cloud (a red band as for sunglint), it is therefore possible to derive an interband calibration. Candidate observations are selected using several criteria: privileged oceanic sites known for their adapted convective dynamics (Fig. 3), very high reflectance level characterizing a strong scattered cloud (up to 0.7), small apparent pressure deducted from the $763 / 765$ ratio [20] assuring a very high altitude of the cloud (less than $400 \mathrm{hPa}$ ), and specific viewing conditions for reduced geometric effects (solar and viewing zenith angles less than $40^{\circ}$ ). Gaseous absorption and correction of the Rayleigh contribution above the cloud is considered. The algorithm includes a multilayer decomposition of the atmosphere and a consideration of the microphysics of the cloud particles: hexagonal columns, plates, or compact hexagonal crystals with a moderated impact on results ( 1 to $2 \%$ depending on wavelength) [21]. Using the red band as reference (as for sunglint), bands from 490 to 865 can be intercalibrated. This method is fully described in Lafrance et al. [21], including the cloud microphysics consideration, i.e., cloud particle type, and applied to POLDER-1 in Hagolle et al. [4].

(d) Cross calibration over desert sites: Desert sites represent remarkably stable targets for which it is possible to perform multitemporal survey and cross calibration with other sensors. 20 desert sites of $100 \times 100 \mathrm{~km}^{2}$, and located in Africa and Arabia (see Fig. 3), were selected for their properties, mainly homogeneity and stability with time [22]. In addition, this temporal stability of such sites can be used to cross calibrate different sensors for which viewing geometries are different [23]. The algorithm researches similar viewing geometries between acquisitions of a given sensor to calibrate and an archive of acquisitions made by a sensor used as reference. The number of such coincidence can be sensitively increased when considering reciprocal viewing conditions [23]. Firstly, leaving from the TOA signal measured by the reference sensor, an appropriate atmospheric correction is made considering molecular and aerosol contributions (desertic aerosol model with an optical thickness of 0.2). A spectral interpolation of the surface reflectance deducted from the reference sensor measurements is made to compute the surface reflectance observed by the sensor to be calibrated weighted by its own instrumental response. Finally, the atmospheric contribution is added to rebuild the TOA signal which is compared to the TOA signal really measured by the sensor to calibrate and for which a typical aspect is given Fig. 2 . This algorithm using acquisitions over 20 desert sites is described in Cabot et al. [23], applied to POLDER-1 in Hagolle et al. [4], and used for ocean color multisensor cross calibration in Fougnie et al. [24].

For all these methods, gaseous corrections were performed using the SMAC approach described in Ref. 25. Gaseous contributions were corrected using exogenous data from meteorological products: ozone content, surface pressure (for oxygen correction, except for calibration over high clouds for which the apparent pressure deducted from 763/765 is used according Ref. 4) and water vapor content.

\section{Calibration Results}

The in-flight calibration of PARASOL was conducted in a similar way as for POLDER [4] or VEGETATION [18] and described in Table 4. In this approach, the ratio of measured radiance, $M I$, to calculated radiance, $C I$, computed using various methods is called $\Delta A_{k}$ because it can be interpreted as a calibration error on the analyzed data for the spectral band $k$. Firstly, an absolute calibration of shorter wavelengths was made using Rayleigh scattering deriving $\Delta A_{k}^{\text {Ray }}(490), \Delta A_{k}^{\text {Ray }}(565)$, and $\Delta A_{k}^{\text {Ray }}(670)$. Secondly, an interband calibration over sunglint using band 670 as reference provided coefficients $\Delta A_{k}^{\text {Sun }}\left(\lambda_{\text {NIR }}\right)$ $=\mathrm{d} A_{k}\left(\lambda_{\mathrm{NIR}}\right) \times \Delta A_{k}^{\text {Ray }}(670)$ for near infrared bands $\lambda_{\mathrm{NIR}}$ where $\mathrm{d} A_{k}\left(\lambda_{\mathrm{NIR}}\right)$ are interband calibration coefficients and assuming $\mathrm{d} A_{k}(670)=1$. The calibration over sunglint also evaluates $\Delta A_{k}^{\text {Sun }}(490)$ and $\Delta A_{k}^{\text {Sun }}(565)$ coefficients for shorter wavelengths. In order to avoid a direct propagation of any bias uniquely due to error on the knowledge of the real $\Delta A_{k}(670)$ to other longer wavelengths, a compromise is found adjusting previous results with $F_{\text {adj }}$ defined by

$$
\begin{aligned}
F_{\text {adj }}= & {\left[\Delta A_{k}^{\text {Ray }}(490)+\Delta A_{k}^{\text {Ray }}(565)+\Delta A_{k}^{\text {Ray }}(670)\right] / } \\
& {\left[\Delta A_{k}^{\text {Sun }}(490)+\Delta A_{k}^{\text {Sun }}(565)+\Delta A_{k}^{\text {Sun }}(670)\right] . }
\end{aligned}
$$

Finally, "compromised" calibration coefficients are defined by

- $\Delta A_{k}^{\text {Ray }}(490), \Delta A_{k}^{\text {Ray }}(565), \Delta A_{k}^{\text {Ray }}$ (670) for visible bands,

- $\Delta A_{k}{ }_{\text {Sun }}\left(\lambda_{\mathrm{NIR}}\right)=F_{\text {adj }} \times \mathrm{d} A_{k}\left(\lambda_{\mathrm{NIR}}\right) \times \Delta A_{k}^{\text {Ray }}(670)$ for NIR bands 765, 865, and 1020.

Note that in absolute calibration over Rayleigh scattering, the band 865 used to correct the residual aerosol background need to be well calibrated. Consequently, if $\Delta A_{k}^{\text {Sun }}(865)$ is found to be sensitively different than 1.0, an iteration is required: the absolute calibration over Rayleigh scattering must be recomputed using $M I(865) / \Delta A_{k}{ }^{\operatorname{Sun}}(865)$ instead of $M I(865)$ to estimate the aerosol radiance, then the interband calibration must be reappraised, and so on ... . Since a $5 \%$ error on band 865 leads to a $1 \%$ error on $\Delta A_{k}^{\text {Ray }}(490)[4,13]$, a good convergence is observed after one or 2 steps.

Results illustrated in Figs. 4-7 for bands 490, 670, and 865 , and summarized in Table 3 , are based on 3 

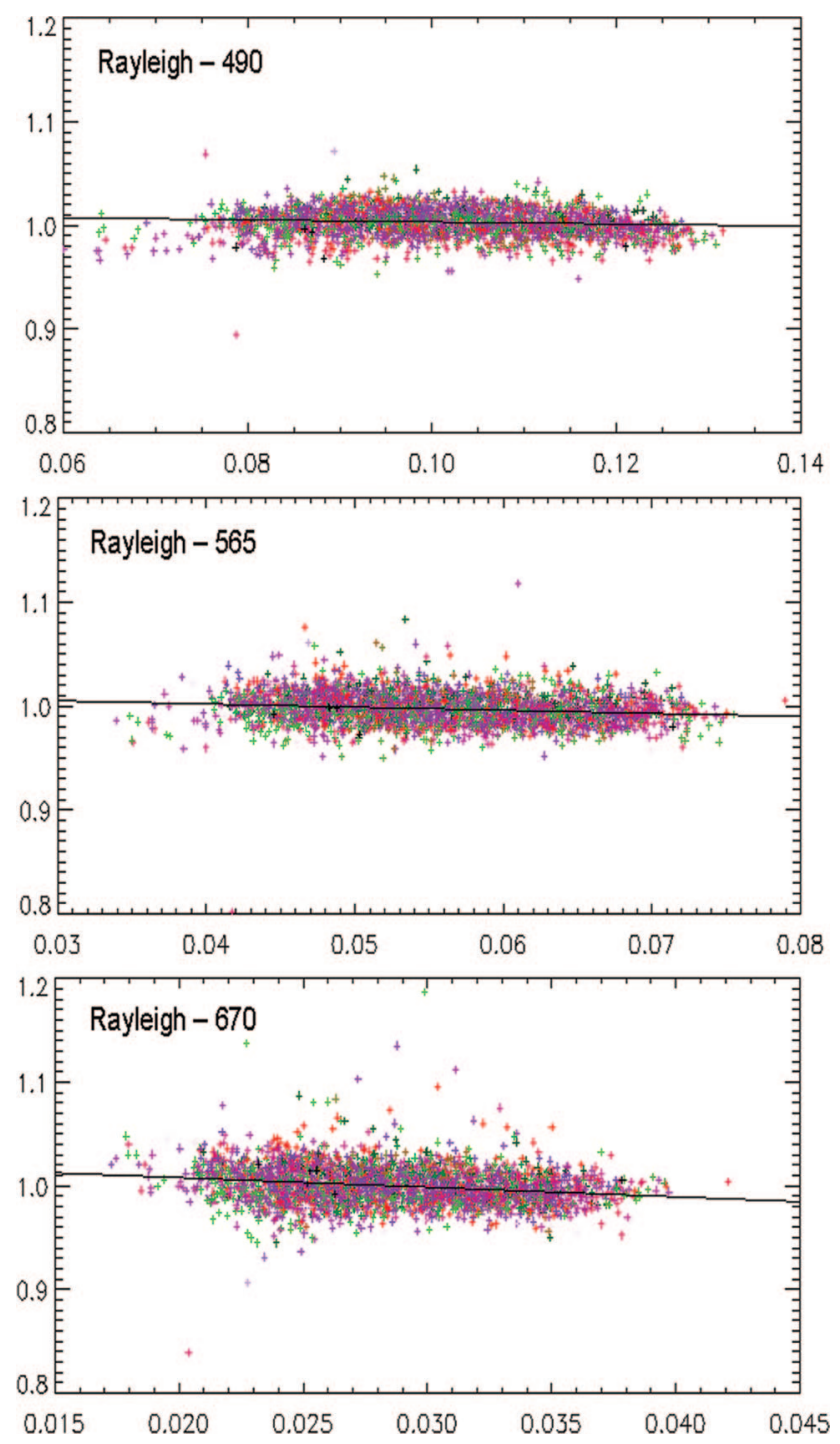

Fig. 4. (Color online) Calibration over Rayleigh scattering. Measured to estimated radiance ratio $\Delta A_{k}$ as a function of the estimated normalized radiance for spectral bands of 490 (top), 565 (middle), and 670 (bottom). Standard deviations are $1.15 \%$ for 490 , $1.38 \%$ for 565 , and $2.29 \%$ for 670 with $N=4457$ points from March to May 2005.

months of PARASOL data from March until May 2005. A strong adjustment from preflight calibration was necessary and the $4 \%$ to $9 \%$, depending on the wavelength, were retrospectively explained by a biased calibration of the integrating sphere used for PARASOL preflight absolute calibration. Results obtained using calibration over Rayleigh scattering (Fig. 4), interband over sunglint (Fig. 5) or over clouds (Fig. 6), and cross calibration with POLDER-2 (Fig. 7) show confident behavior when analyzed as a function of the observed normalized radiance (as in Figs. 4-7) or other geometric or geophysical parameters. Figure 8 and Table 3 point out the good global consistency between results for all the spectral range (except the band 443): results are within $\pm 1 \%$ for 490 and 670 , $\pm 1.5 \%$ for 865 , and $\pm 2 \%$ for 565,765 , and 1020 . Such a consistence from various calibration methods us-
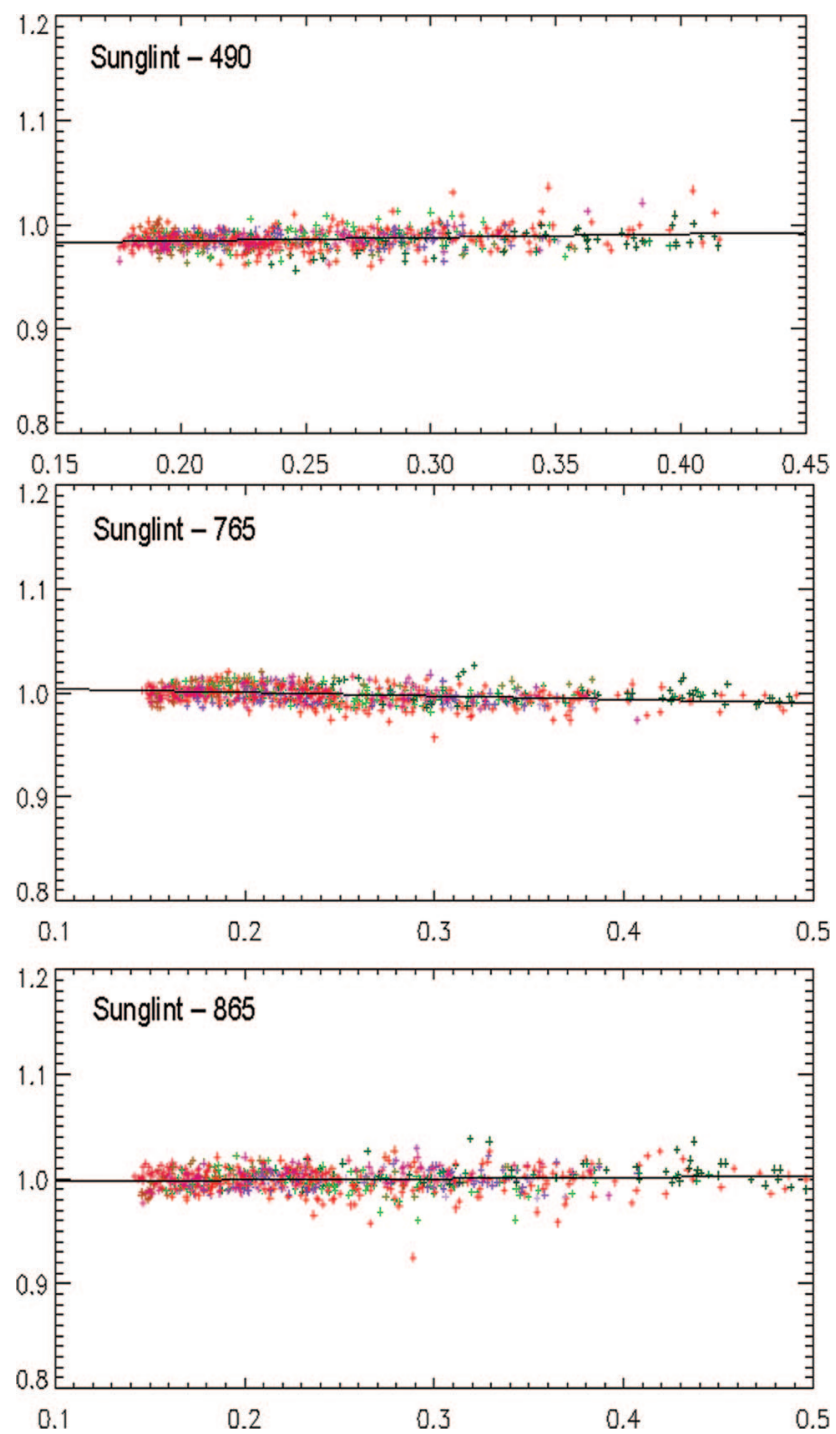

Fig. 5. (Color online) Interband calibration over sunglint. Measured to estimated radiance ratio $\Delta A_{k}$ as a function of the estimated normalized radiance for spectral bands of 490 (top), 765 (middle), and 865 (bottom). Standard deviations are $0.88 \%$ for 490 , $0.84 \%$ for 765 , and $1.05 \%$ for 865 with $N=765$ points from March to May 2005.

ing four different targets corresponding to very different spectral signatures and reflectance levels (as shown in Fig. 2), and very varied geometric conditions (viewing and solar), gives a good confidence on the PARASOL level-1 calibration.

The calibration of the band 763 , centered on the oxygen A-band absorption, can be derived from the absolute calibration of the band 765 , which was adjusted by $7 \%$ (see Table 3). The method consists in comparing the $\mathrm{O}_{2}$ transmission obtained using surface pressure at the sea level in clear sky conditions and the transmission derived from the differential absorption method using PARASOL observations at bands 763 and 765 [20]. As described in Ref. 4, acquisitions over sunglint are adequate for this calibration. Results evidenced a necessary $-2 \%$ adjustment on the preflight interband calibration of bands 763 

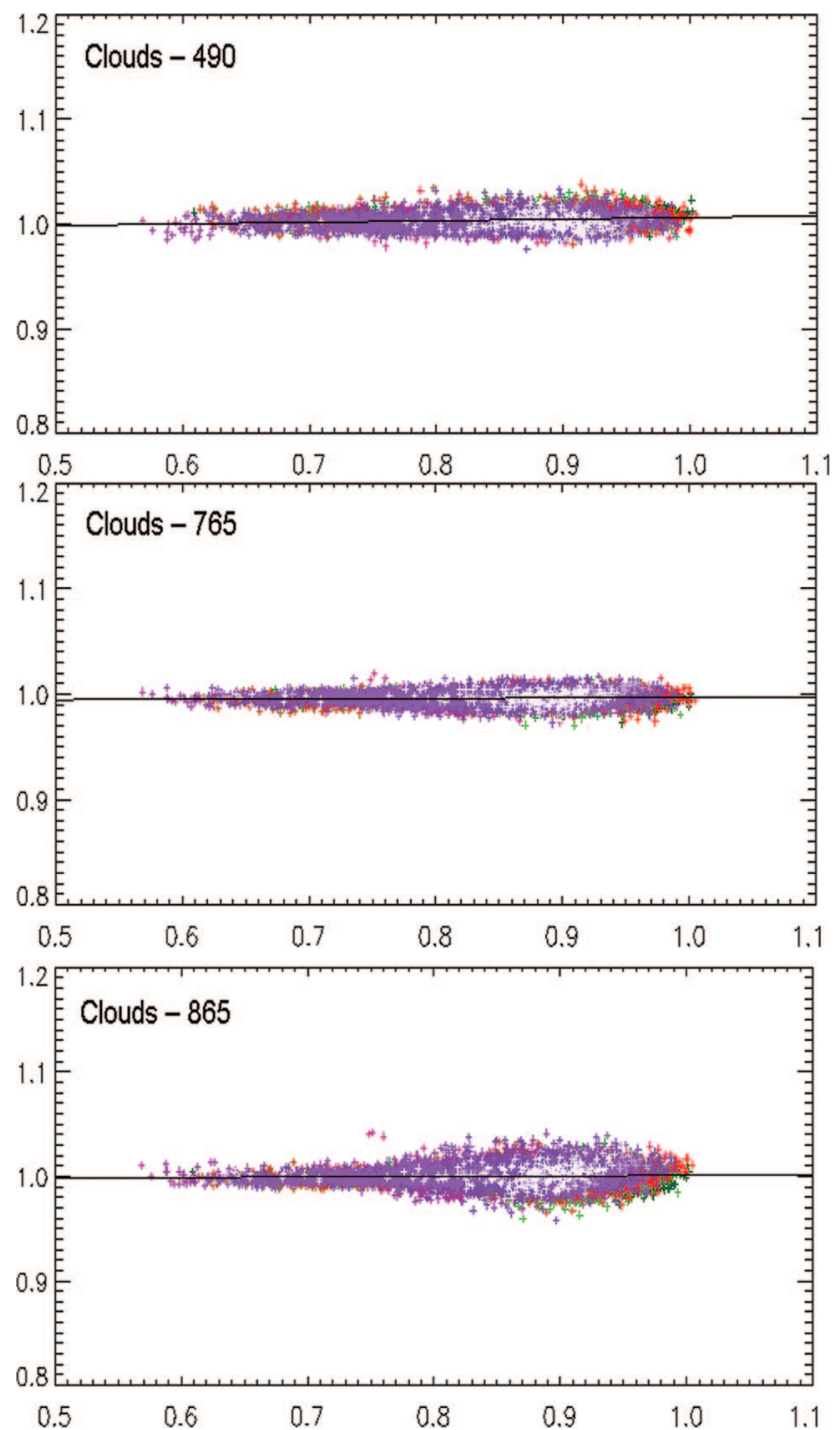

Fig. 6. (Color online) Interband calibration over clouds. Measured to estimated radiance ratio $\Delta A_{k}$ as a function of the estimated normalized radiance for spectral bands of 490 (top), 765 (middle), and 865 (bottom). Standard deviations are $0.67 \%$ for $490,0.54 \%$ for 765 , and $0.87 \%$ for 865 with $N=10614$ points from March to May 2005.

and 765 leading to an absolute adjustment for 763 of $5 \%$ (Table 3). The band 910 is centered on a water vapor absorption peak. The same approach is used than for band 763 , i.e., band 910 is intercalibrated with band 865 . This intercalibration between bands 910 and 865 was kept to preflight calibration as it was made for previous POLDER 1 and 2 [4].

\section{Multitemporal Monitoring}

Evolution with time of the radiometric sensitivity of the instrument is a natural process. During the first days or months in orbit, some optical parts of the instrument may have molecular outgassing phenomena in the vacuum of space. A more or less long-term effect evolution may be due to aging of the optical parts enduring "aggressive" solar irradiation. Several space sensors are equipped with on-board device to monitor
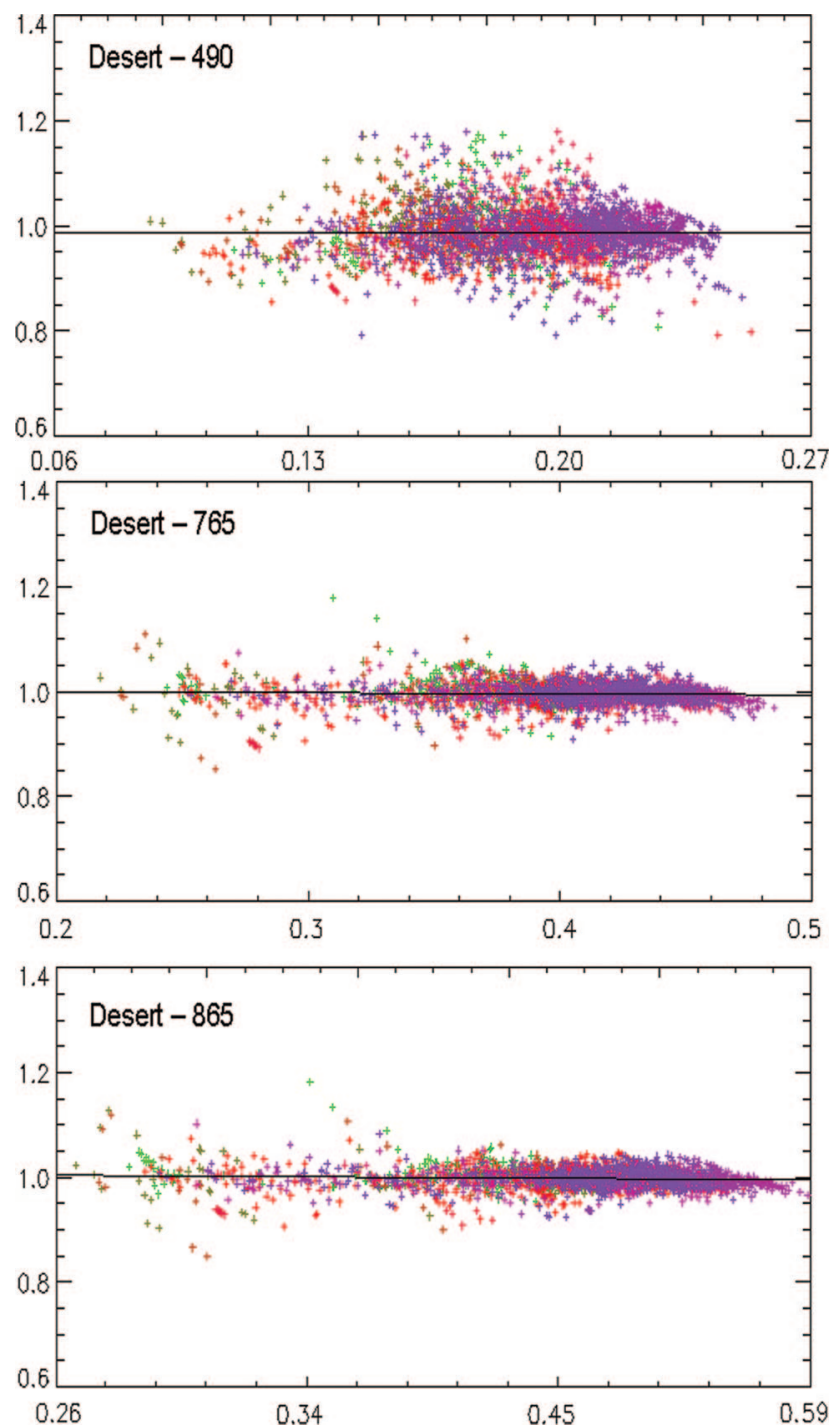

Fig. 7. (Color online) Calibration over desert sites. Measured to estimated radiance ratio $\Delta A_{k}$ as a function of the estimated normalized radiance for spectral bands of 490 (top), 765 (middle), and 865 (bottom) using POLDER-2/ADEOS-2 as reference. Standard deviations are $4.61 \%$ for $490,2.13 \%$ for 565 , and $2.05 \%$ for 670 with $N=3941$ points from March 2005 to March 2006 .

possible temporal evolution. SeaWiFS [26], MERIS [27], or MODIS [28] are equipped with solar diffusers while Vegetation/SPOT [18] is equipped with an onboard lamp. In all cases, difficulties are encountered: aging of the lamp or diffuser themselves may occur requiring a complementary on-board monitoring through diffuser duplication for example [27,28]. Complementarily, the moon is used as a natural and external diffuser to monitor SeaWiFS long-term trends using acquisitions for specific lunar phases [29]. Alternative methods using natural targets from the Earth-atmosphere system were developed: over desert sites [23,24], viewing sunglint [18] or over Antarctica [30]. Nevertheless, some limitations are due to perturbing contribution or inaccuracy on the algorithm, such as aerosol perturbation, seasonal effects, bidirectional effects, spectral behavior 
Table 3. Summary of the Calibration Results $A_{k}$ for Rayleigh, Sunglint, Clouds, and Deserts Methods ${ }^{a}$

\begin{tabular}{llllllllll}
\hline & \multicolumn{9}{c}{ Spectral Band } \\
\cline { 2 - 7 } & 443 & 490 & 565 & 670 & 763 & 765 & 865 & 910 & 1020 \\
\hline Preflight & 1.000 & 0.959 & 0.964 & 0.947 & 0.950 & 0.930 & 0.926 & 0.926 \\
Rayleigh & 0.899 & 1.002 & 0.999 & 1.001 & & & & \\
Sunglint & 0.960 & 0.994 & 0.997 & 1.008 & 1.005 & 1.005 & 1.008 & \\
Clouds & 1.047 & 0.999 & 1.006 & 0.995 & & 0.984 & 0.984 & 0.999 \\
Deserts & 0.970 & 0.998 & 1.026 & 0.996 & & 1.018 & 0.999 \\
\%/year & -4.05 & -2.98 & -1.70 & -0.99 & 0.00 & 0.00 & -0.40 & -0.40 \\
\hline
\end{tabular}

${ }^{a}$ When the computed radiance is in accordance with the measured radiance, $A_{k}=1.0$. The interband calibration results (sunglint and clouds methods) are reported after a normalization to $\left[A_{k}(490)+A_{k}(565)+A_{k}(670)\right] / 3$. The preflight calibration values are also reported. The temporal drift of the radiometric sensitivity is calculated in $\%$ per year.

modeling, or difficulty to obtain a dense temporal sampling.

PARASOL is not equipped with an onboard calibration device. After one year in orbit, it was evidenced by all methods used to estimate the absolute calibration (Subsection 2.B.1) that a temporal decrease of the radiometric sensitivity occurred. It was necessary to choose the best reference as possible to monitor the multitemporal decrease of the sensitivity. Regarding theoretical error budgets for each method [4,18,21,23,31], effective behaviors of results (bias and root mean square error), spectral shapes of the targets leading to potential errors into the model, and finally the temporal sampling, the calibration over white convective clouds was preferred as the best compromise. In addition, this reference is physically very close to the white moon diffuser that provided essential results for SeaWiFs [29].

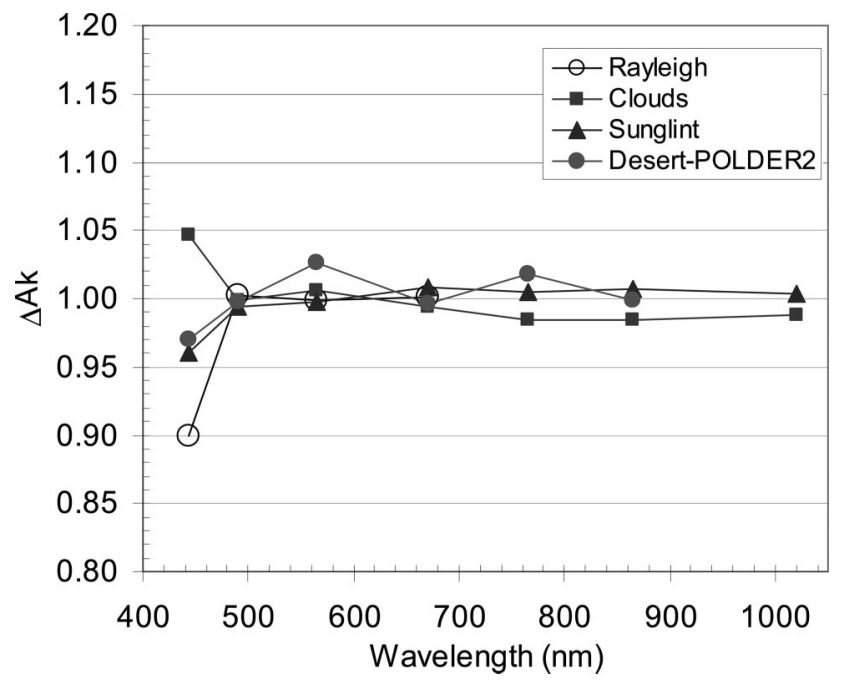

Fig. 8. Synthesis of PARASOL in-flight calibration results. Measured to estimated radiance ratio $\Delta A_{k}$ for all PARASOL spectral bands and for calibration over Rayleigh scattering (circle), interband over sunglint (triangle), interband over clouds (square) and over desert sites using POLDER-2 as a reference (dot). Interband results (clouds and sunglint) reported here are renormalized to the mean of 490-565- 670 values to be comparable with results from the two other absolute methods.
Figures 9(a)-9(c) measurements were analyzed over 18 months for the four calibration methods and for bands 490, 670, and 865 separately. For interband methods, i.e., calibration over sunglint and clouds, a temporally stable reference was necessary to derive results presented in Fig. 9: The band 765 was selected as the best stable reference with time. This last hypothesis is based on two arguments: First, interband results show that all the spectral bands are decreasing more with time than band 765 [Fig. 10(a)] Second, when the band 765 is supposed stable, a very good consistency is found with absolute calibration over Rayleigh scattering and cross calibration over desert sites, for all spectral bands (Fig. 9) including the band 765. A very good consistency is observed for red (670) to near-infrared (865) spectral bands: The temporal drift is found to be sensible for bands 490 , 670 , and a very small for band 865 . Results over sunglint or desert sites for the band 490 seem inaccurate with a strong dispersion as confirmed by theoretical error budgets [18,23,31]. Multitemporal calibration over Rayleigh scattering provides interesting confident results for bands 490 and 670, but the best repeatability is observed for white convective clouds, confirming the adequation of such diffusers as excellent references. Figures 9 and 10 show the annual mean drift can be estimated with an accuracy within $0.5 \%$ per year. Figure 10(a), results of calibration over clouds, are shown for all spectral bands (except gaseous and 443 bands). The temporal drift in $\%$ per year varies from $-3 \%$ at 490 to $-1 \%$ at 670 , is null for 765 , and reached $-1.5 \%$ for 1020 . Exponential functions are used for SeaWiFs [26] and MERIS [27] to model the temporal drift, but Fig. 10(a) shows that a linear model is sufficient to describe the PARASOL evolution. The prediction model illustrated Fig. 10(b) is based on a linear fit over the 15 first months, from March 2005 until June 2006. Since July 2006, the prediction model is used to operationally predict the new calibration coefficient to be applicable in the level-1 processing of the next month: for this, the linear regression is updated based on the measurement dataset completed by the new measurement of the current month. A long term monitoring will deal with the two assumptions that the 

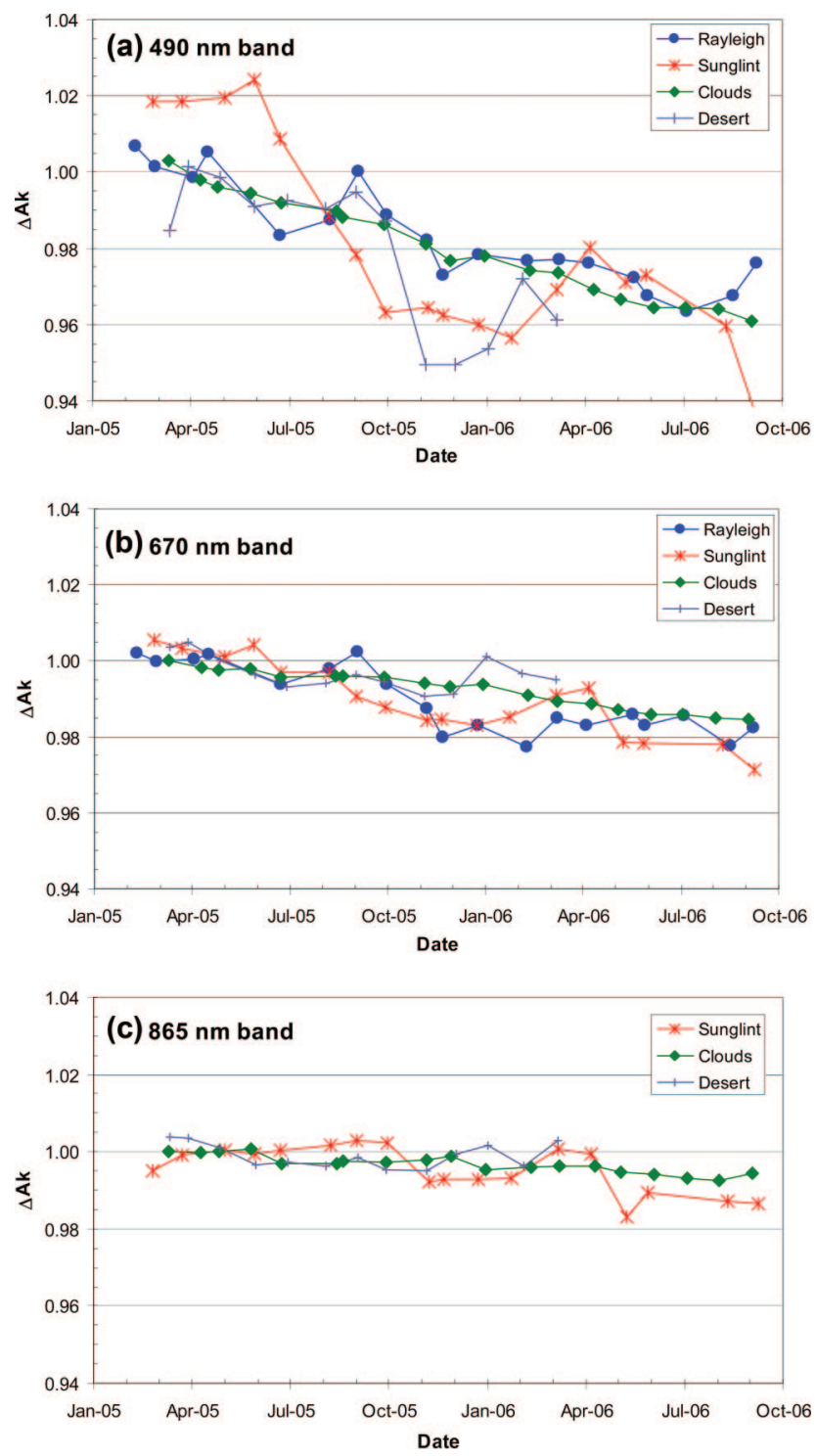

Fig. 9. (Color online) Temporal evolution of $\Delta A_{k}$ from March 2005 to September 2006 for bands 490 (a), 670 (b), and 865 (c) as observed for various calibration methods. For one given spectral band and calibration method, the set of measurements is normalized to a reference value computed for 1 April 2005 using a linear regression on this set. For interband methods, the band 765 is used as reference and is supposed to be stable with time.

evolution can be assimilated to a linear model and that the band 765 is stable with time. As for absolute calibration, the oxygen band 763 and water vapor band 910 are intercalibrated with bands 765 and 865 respectively (see Table 4).

\section{Other Radiometric Characterization}

\section{Dynamic Over the Orbit}

The instrument was designed for observation of clouds and aerosols. In particular, and according to the mission requirements, the saturation level of the instrument has been adjusted to a TOA normalized radiance of 1.0 through the preflight instrumental design, but also an in-flight readjustment of integra-
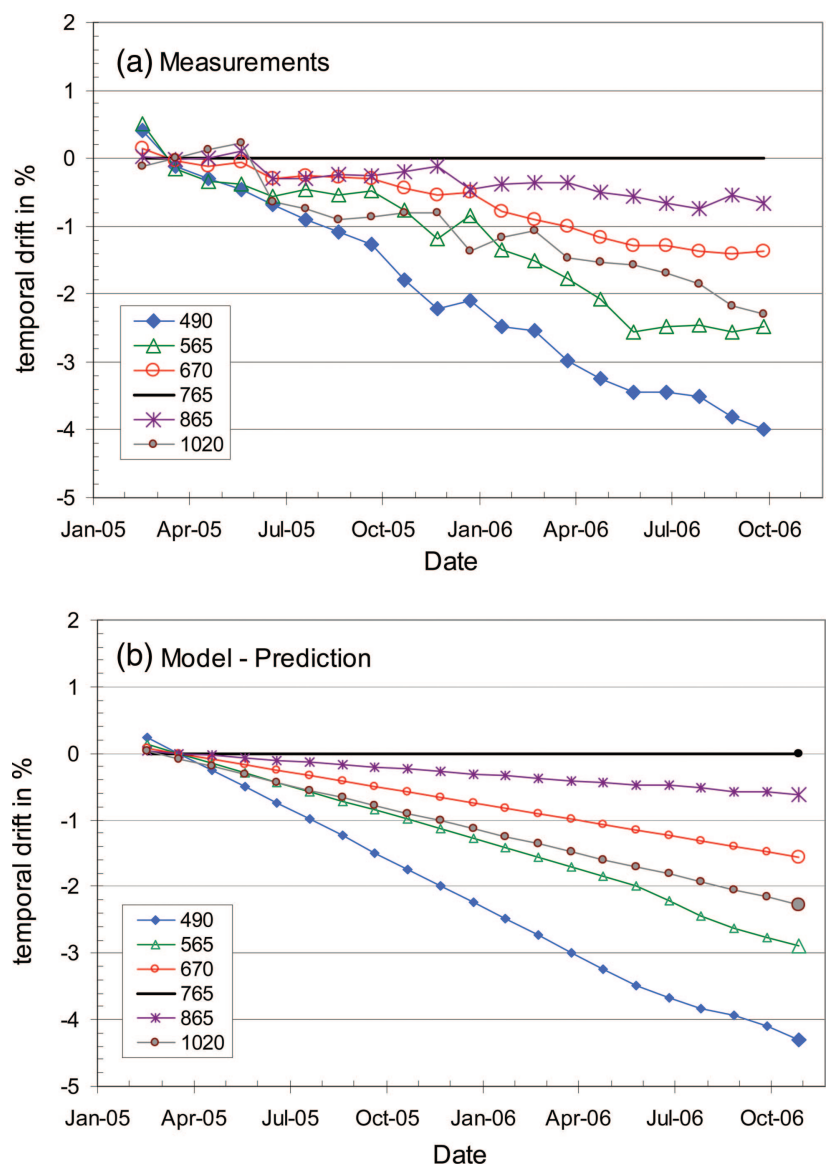

Fig. 10. (Color online) Temporal drift in \% of the radiometric sensitivity as measured over clouds [(a), top] and modeled [(b), bottom]. (a) The temporal drift is reported as observed from March 2005 to October 2006 over scattered convective clouds for viewing and solar angles less than $40^{\circ}$ ("nadir" conditions). Results are normalized for 1 April 2005 and standard deviations are $1.14 \%$ for $490,0.80 \%$ for $565,0.57 \%$ for $670,0.66 \%$ for 865 , and $2.83 \%$ for 1020. The band 765 is used as reference. (b) The temporal drift is reported as estimated by the prediction model. The drift is approximated by a linear regression from March 2005 to May 2006 (initialization of the model). Since then the linear regression is updated each month, from June to November 2006, to operationally predict the temporal drift. Big symbols are the calibration coefficients-predicted for November 2006 using the measurements from March 2005 until October 2006 presented in (a).

tion times. Considering that two integration times are possible when programming the instrumental acquisition of the 9 spectral bands (short and long integration times SIT and LIT respectively [1]), the optimization was made globally for the entire set of spectral bands. Table 2 shows the real saturation level obtained for each spectral band after in-flight calibration and the global optimization of integration times: Values are close to or higher than 1.0 avoiding saturation over bright clouds. Complementarily, the ability to vary the integration time along the orbit was allowed to the PARASOL instrument. For each orbit, the integration time progressively increases from equator to poles using a $\cos ^{-1}\left(\theta_{S}{ }^{\min }\right)$ variation law where $\theta_{S}{ }^{\mathrm{min}}$ is the minimum zenith solar angle of 
Table 4. Summary of Methods Used to Calibration all PARASOL Spectral Bands for Both Absolute and Temporal Aspects ${ }^{a}$

\begin{tabular}{|c|c|c|c|c|c|c|c|c|c|}
\hline Calibration & Method & \multicolumn{8}{|c|}{ Spectral Band } \\
\hline Absolute & Rayleigh (ray) & ray & ray & ray & & & & & \\
\hline Multitemporal & Clouds (cld) & cld & cld & cld & 765 & ref. & cld & 865 & cld \\
\hline
\end{tabular}

${ }^{a}$ Methods are absolute calibration over Rayleigh scattering (ray), interband calibration over sunglint (sun), and multitemporal calibration over convective clouds (cld). "ref" means that spectral bands are used as reference (temporal or absolute) to calibrate other spectral bands. When 765 or 865 is reported, this band is used a reference for inter-calibration (for 763 or 910 respectively).

all ground points instantaneously observed inside a given PARASOL image. In other words, the instrumental dynamic is readjusted for each image to take into account the decreasing solar irradiance along the orbit, and consequently, the saturation level can be expressed as 1.0 in reflectance unit (not only normalized radiance unit).

\section{In-Polarization Calibration}

The POLDER instrument is able to measure the polarization for three spectral bands, 490, 670, and 865 . For each of these bands, three measurements are made through a polarizer with three different orientations, and the Stokes vector $[I, Q, U]$ can be easily derived according the radiometric model inversion as shown in Ref. 5. Wide field-of-view optics like POLDER instruments have a small sensitivity to the polarization state of the observed light. In other words, the optics can be seen as a polarizer characterized by a very poor efficiency, called $\varepsilon$, varying from $0 \%$ at nadir to typically $3 \%-4 \%$ for large viewing angles as illustrated in Figs. 1 and 11. This sensitivity is fully characterized before launch using an appropriate set of polarized measurements, but it is nevertheless necessary to check after launch this parameter $\varepsilon$. Thus, a methodology has been developed using specific acquisitions selected for their theoretical unpolarized signatures. The polarized intensity Ip defined by the square root of $\left(Q^{2}+U^{2}\right)$, where $Q$ and $U$ are Stokes parameters, is analyzed and for

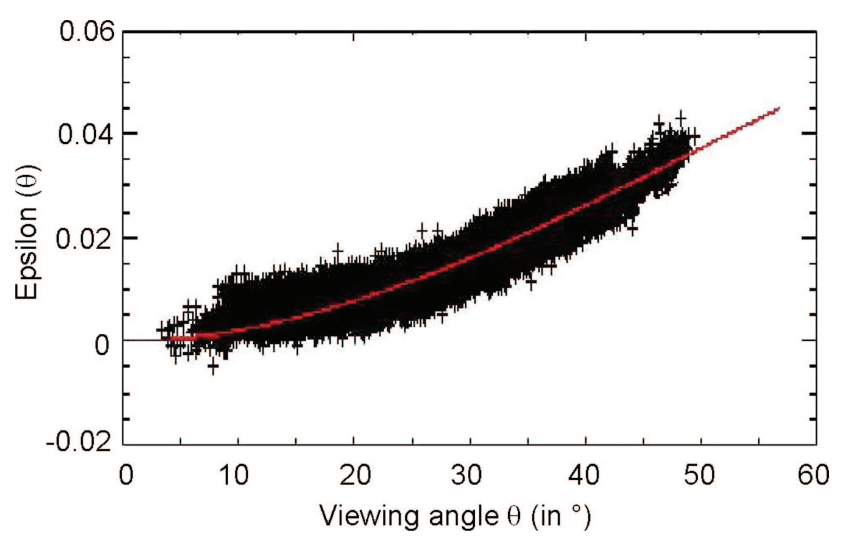

Fig. 11. (Color online) In-flight estimation of the polarization sensitivity of the instrument for the band $670, \varepsilon(\theta)$, based on measurements over clouds in specific geometries for which the observed target is unpolarized. such selected acquisitions, Ip derived by the radiometric model inversion is expected to be null if $\varepsilon$ is well calibrated. Signal observed over bright scattered clouds (targets used for the interband calibration) for a specific scattering angle near $160^{\circ}$ is unpolarized whatever the microphysics of the clouds: ice crystal or water droplet particles [32]. Assuming that the polarizer efficiency $\eta$ (see Subsection 2.A) is perfectly known from preflight characterization, this property was used as illustrated in Fig. 11 to estimate the $\varepsilon$ function for the band 670 with a very good similarity with the preflight function given in Fig. 1. Furthermore, the $\varepsilon$ function was validated with a relative accuracy close to $10 \%$ for the three polarized bands, 490,670 , and 865 and we can conclude that no significant evolution of the polarized sensitivity of the optic occurred. This conclusion can be extrapolated to all PARASOL spectral bands, i.e., the six other unpolarized bands, because the sensitivity to polarization, by construction due to the fact that incident beams for large viewing angles are not perfectly normal to all lenses when going through the optic, is a phenomenon slowly varying with wavelength.

\section{Nonlinearity Function}

The nonlinearity behavior of POLDER instruments was evidenced on POLDER-1 in-flight data using the redundancy of 443 polarized and unpolarized bands [9]. For PARASOL, this function was very carefully characterized with dedicated preflight sets of measurements exploring the entire dynamic range of the instrument. As shown in Table 2, PARASOL spectral bands have been slightly modified compared to POLDER, particularly the loss of the band 443 redundancy which is now only an unpolarized spectral band. Consequently, an other in-flight method has been developed but the principle remains in the comparison of two observations of the same reflectance but acquired for very different integration times.

Specific programmings were realized in the first two months of the commissioning phase during which it was possible to alternate every 20 seconds a very short integration time acquisitions (SIT of $10 \mathrm{~ms}$ ) and a very long integration time acquisition (LIT of $62 \mathrm{~ms}$ ). For these experimental segments, the nonlinearity correction defined in Fougnie et al. [9] was ignored on the level-1 data processing. The multidirectional ability of PARASOL allows us to select for the same white target, two viewing directions near the nadir viewing and acquired successively with SIT 


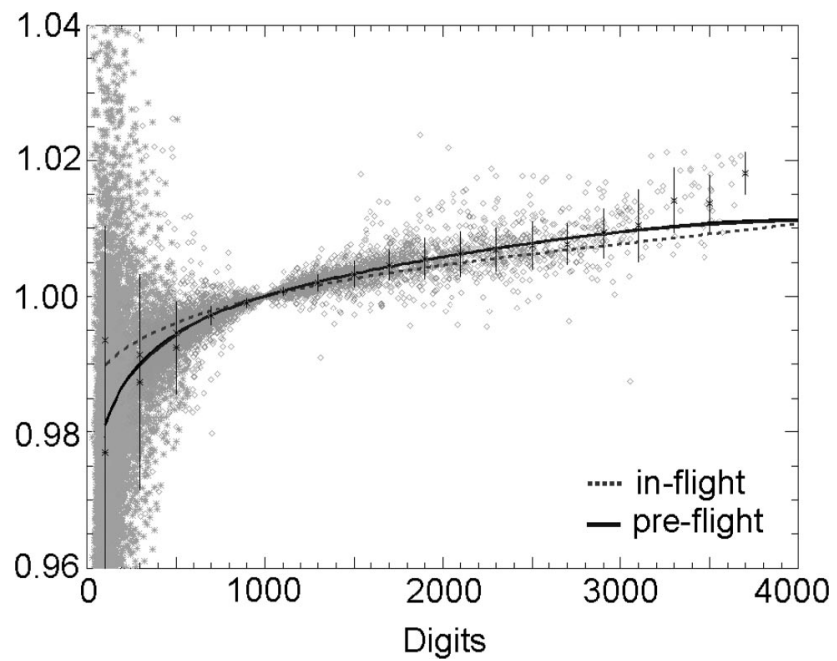

Fig. 12. Nonlinearity function as defined by Fougnie et al. [9], derived from preflight characterization (plain line), and measured in-flight using SIT/LIT alternated acquisitions (diamonds). The in-flight estimated model (dashed line) is consistent with preflight model within $0.5 \%$. All estimations have been normalized to 1.0 for digit 1000 .

and LIT. In this conditions, a couple of points is defined for a given observation by $X_{S}$ and $X_{L}$, digits measured for SIT and LIT respectively. In order to allow concatenation of each couple and comparison with preflight function, a normalization must be made for the reference digit 1000 . The equivalent integration time for digit 1000 is computed according $t_{1000}=\mathrm{SIT}+(\mathrm{LIT}-\mathrm{SIT}) /\left(X_{L}-X_{S}\right) \cdot\left(1000-X_{S}\right)$. Then $\left(X_{S} / \mathrm{SIT}\right) .\left(t_{1000} / 1000\right)$ and $\left(X_{L} / \mathrm{LIT}\right) .\left(t_{1000} / 1000\right)$ ratios are computed for each couple of measurements, reported on Fig. 12 as a function of $X_{L}$, and compared to the preflight nonlinearity function. As it is difficult to claim an accuracy better than $0.5 \%$ using this inflight method, the preflight model is considered as validated within $0.5 \%$ except for extremely low digits for which the radiometric noise is obviously limiting.

\section{E. Radiometric Performance}

\section{Multiangular Behavior}

The multiangular behavior of the instrument is given by the product $P(\theta) g_{l, p}$ described in the radiometric model (Subsection 2.A). As shown in Fig. 1, the $P(\theta)$ function is a polynomial and is called low-frequency term. At the opposite, $g_{l, p}$ values are interpixel variations of the sensitivity usually called high-frequency terms [4]. It is not possible to directly assess in-flight the polynomial $P(\theta)$. However, it is possible to carefully check the behavior of all calibration results (presented in Subsection 2.B) as a function of the viewing angle $\theta$ as shown in Fig. 13. No bias was evidenced for all spectral bands by the four calibration methods and the preflight estimation of $P(\theta)$ is considered as validated within $2 \%$ for viewing angles of $50^{\circ}$. Highfrequency terms $g_{1, \mathrm{p}}$ were checked through the statistical method described in Ref. 4 or Ref. 8. In-flight estimation confirms the preflight coefficients with a standard deviation of $0.022 \%$ for blue bands to
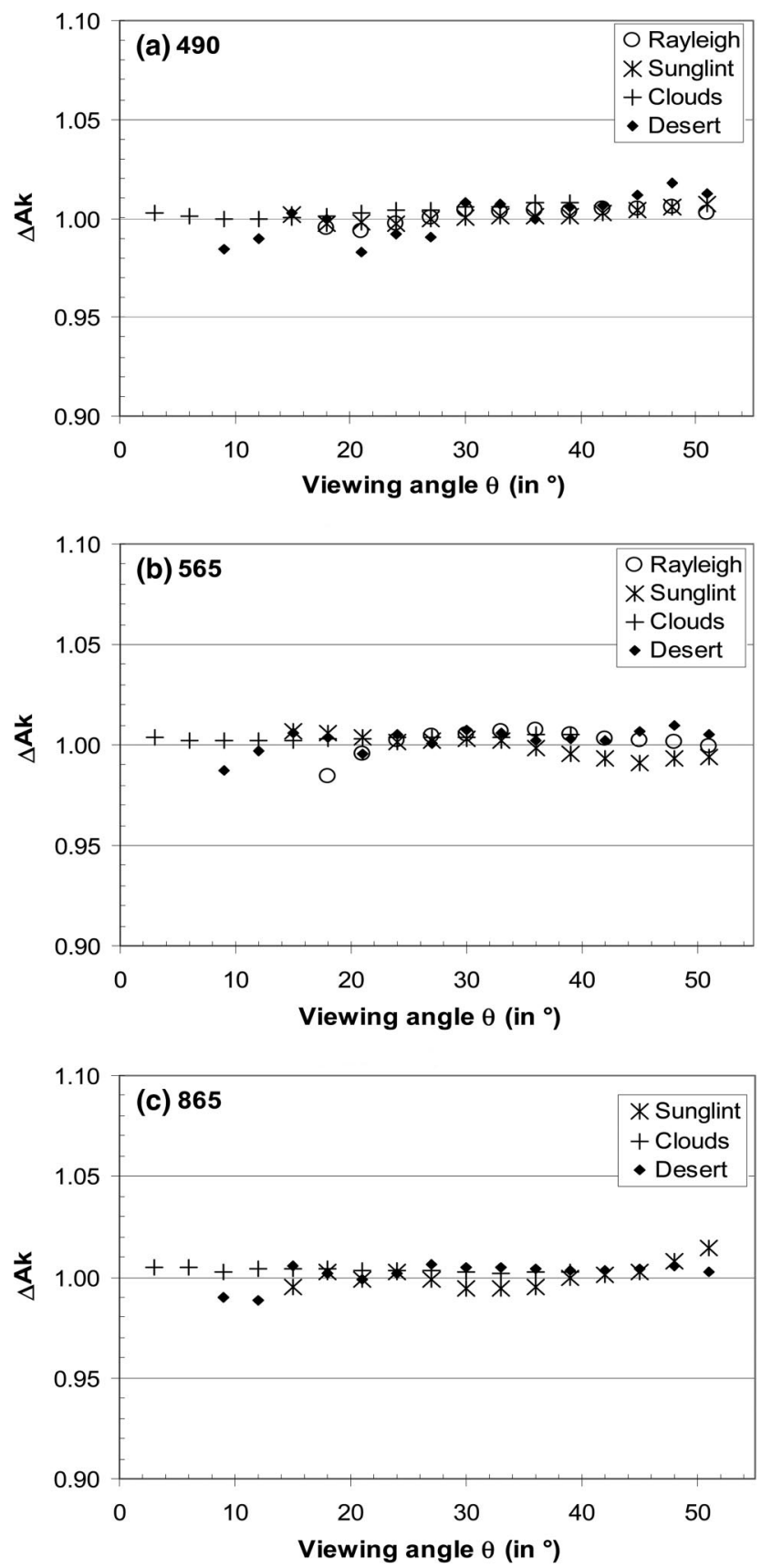

Fig. 13. Calibration results summarized as a function of the viewing angle for the 4 calibration methods. Measured to estimated radiance ratio $\Delta A_{k}$ were averaged every 3 degrees for bands 490 [(a), top], 565 [(b), middle], and 865 [(c), bottom].

$0.016 \%$ for near infrared bands with maximum biases of $1.2 \%$. These marginal biases can be explained by punctual inaccuracy on the preflight estimation or by evolution after launch, such as dust deposited in the frontal lens of the instrument.

\section{Noise Estimation}

The noise equivalent derived level-1 reflectance is a sum of various contributions including random fluctuations of the signal, errors on the knowledge of radiometric model parameters (also called equaliza- 


\begin{tabular}{|c|c|c|c|c|c|c|c|c|}
\hline & & & \multicolumn{6}{|c|}{ Spectral Band } \\
\hline & & & 490 & 565 & 670 & 765 & 865 & 1020 \\
\hline \multirow[t]{3}{*}{ Dark current } & $I=0$ & NedI & 0.00009 & 0.00010 & 0.00009 & 0.00010 & 0.00011 & 0.00018 \\
\hline & & Preflight & 0.00023 & 0.00023 & 0.00023 & 0.00023 & 0.00023 & 0.00023 \\
\hline & & Spec. & 0.00050 & 0.00050 & 0.00050 & 0.00050 & 0.00050 & 0.00050 \\
\hline \multirow[t]{3}{*}{ Dark ocean } & $I \leq 0.1$ & NedI & & & & 0.00047 & 0.00029 & 0.00042 \\
\hline & & Preflight & & & & 0.00025 & 0.00024 & 0.00033 \\
\hline & & Spec. & & & & 0.00050 & 0.00050 & 0.00050 \\
\hline \multirow[t]{3}{*}{ Ocean } & $I p<0.1$ & NedIp & 0.00085 & & 0.00085 & & 0.00042 & \\
\hline & & Preflight & 0.00052 & & 0.00051 & & 0.00050 & \\
\hline & & Spec. & 0.00100 & & 0.00100 & & 0.00100 & \\
\hline \multirow[t]{3}{*}{ Clouds } & $I p \sim 0.1$ & NedIp & 0.00255 & & 0.00325 & & 0.00269 & \\
\hline & & Preflight & 0.00113 & & 0.00267 & & 0.00085 & \\
\hline & & Spec. & 0.00100 & & 0.00100 & & 0.00100 & \\
\hline
\end{tabular}

tion residue), error on the stray-light correction, and error due to the resampling on the level-1 data grid. The absence of onboard calibration device prevents in-flight accurate quantification of the noise level. Moreover, it is impossible to found large enough areas that could be considered as perfectly uniform regarding the PARASOL swath and spatial resolution. Nevertheless, attempts have been made to assess the noise considering that rough estimations are overestimations of the real level-1 data noise because affected by residual nonuniformities of the area or artifacts due to the method.

Standard deviations of the dark current were extracted from raw acquisitions and were converted into noise equivalent to normalized radiance (NedI). As this measurement is made at the detector level, it mainly represents the contribution from electronic fluctuation of the signal, and this is the reason why values are found to be smaller than preflight estimations of noise for extremely low radiance (Table 5). Probably more representative, images were selected over ocean for which no marine structure were identified for near-infrared bands. Dispersion of level-1 normalized radiances was calculated for arbitrary small homogenous areas but inevitably, a residual geophysical variation neglected here is still present inside the area. Table 5, level-1 data noise estimation over dark ocean, i.e., low radiances, are very consistent with preflight estimations and inside specified values.

Following formulation and results shown by Schutgens et al. [33], the Stokes parameter $U$ defined using the single-scattering plane as reference, is null for the principal plane which is defined for a given ground point by the local vertical and the solar incident direction. This physical property was used to derive an estimation of the radiometric noise. The dispersion of $U$ values for pixel observed on the principal plane, i.e., observed under a relative viewing azimuth angle of 0 modulo $\pi$, were calculated and converted into noise equivalent to polarized normalized radiance
(NedIp). Table 5 shows results for ocean targets (low polarized reflectance) and cloud targets (moderated polarized reflectance): If values are consistent with preflight estimation and specification for ocean surfaces, it is not the case for clouds. For such heterogeneous targets, the preflight error budget has shown a large radiometric impact of the onboard registration of polarized triplets (mainly for the band 670, see Subsection 3.C.3) which is by construction not perfect. In addition, onboard and level-1 geometric registration are optimized for the ground level and consequently, clouds usually located at various altitude, typically from 0 to $10 \mathrm{~km}$, are naturally misregistrated. The result shown here is clearly an overestimation of the real performance and it has been shown by a similar processing, that performances are completely comparable to POLDER-2 data performance and so acceptable for scientific applications.

\section{Geometric Calibration and Performance}

\section{A. Preflight Characterization}

For a given spectral band $k$, the geometric model describing the exact viewing angle $\theta$ for each pixel $P$ of the image, i.e., the CCD matrix, is written

$$
S P=f_{1}^{k} \tan (\theta)+f_{3}^{k} \tan ^{3}(\theta)+f_{5}^{k} \tan ^{5}(\theta),
$$

where $S$ is the position of the optical center on the CCD matrix, and $f_{1}^{k}, f_{3}^{k}$, and $f_{5}^{k}$ are polynomial functions characterizing the optical distortion of the instrument. Optical center, focal and distortion parameters of the model were derivate for each spectral band during a preflight campaign using an optical reference generator equipped with 29 collimated lamps [6]. Complementarily, an accurate measurement of the alignment of the camera to the platform reference was made before launch to initialize the calibration parameters (Table 6) necessary for the geometrical location into the level-1 data grid [5]. 
Table 6. In-Flight Geometrical Calibration of the Alignment Matrix (Roll, Pitch, and Yaw) of the Instrument in Microradians ${ }^{a}$

\begin{tabular}{lrrr}
\hline $\begin{array}{c}\text { Measure } \\
\text { (in microradian) }\end{array}$ & \multicolumn{1}{c}{ Roll } & \multicolumn{1}{c}{ Pitch } & \multicolumn{1}{c}{ Yaw } \\
\hline Initial calibration & -1176.3 & -1023.1 & -151.6 \\
Iteration 1 & -429.9 & -957.7 & -481.9 \\
Stdev & 117.2 & 39.3 & 63.8 \\
Iter1-initial & 746.4 & 65.4 & -330.3 \\
& & & \\
Iteration 2 & -317.2 & -955.1 & -521.7 \\
Stdev & 103.2 & 42.1 & 48.9 \\
Iter2-iter1 & 112.7 & 2.6 & -39.8 \\
\hline
\end{tabular}

${ }^{a}$ Mean and standard deviation values found for the two iterative steps using space triangulation algorithm are compared to the initial preflight calibration.

\section{B. Absolute Calibration}

The in-flight geometric calibration algorithm is a threefold method. First, a correlation algorithm searches homologue points between images corresponding to the different viewing angles of a given point at the ground. If everything was perfect, all identified points should be exactly superposed, but it is not the case because of residual errors on the geometrical model knowledge, satellite attitude, but also mainly for alignment errors which can be also seen as a satellite attitude bias. For a better accuracy, images mostly free of clouds and containing specific ground points such as ragged coastlines and large lakes are privileged. Secondly, a space triangulation algorithm is used to estimate the satellite attitude bias that minimizes the root mean square superposition error for the set of homologue points previously generated. Third, test segments are generated with the new proposed calibration correcting the estimated attitude bias, and a multiangular registration performance, very sensitive to calibration error (see Subsection 3.C.1), is computed to confirm the improvement on data quality. As the error made on the estimated calibration bias is depending on the magnitude of this bias, an iterative process is usually required to an optimum performance of the algorithm. A fast convergence was found for PARASOL because only 2 iterative steps were necessary as shown in Table 6: The improvement between iteration 2 and 1 is within the standard deviation of the result. The band 765 was used to geometrically calibrate the instrument, but the independence to wavelength was punctually verified for the band 565. Finally, no variation with latitude, signature for a possible thermoelastic deformation of the structure, was evidenced.

\section{Geometric Performances}

Geometrical registration performances are estimated using correlation algorithms. The CCD matrix was divided into $11 \times 10$ areas for a better analysis of residual signatures. In addition, this area-by-area approach limits the impact of unfortunate wrong correlations and allows a better filtering of all measurements to elaborate the general result. These performances were established during the commissioning phase in spring 2005 and were re-evaluated in spring 2006 . No evolution was detected on geometrical calibration and performances. In a general way, all specifications are applicable for viewing zenith angle within $50^{\circ}$.

\section{Multiangular Registration}

The multidirectional ability of PARASOL is due to its very wide field-of-view: One point at the ground is seen $N$ times under different viewing angles, $N$ being
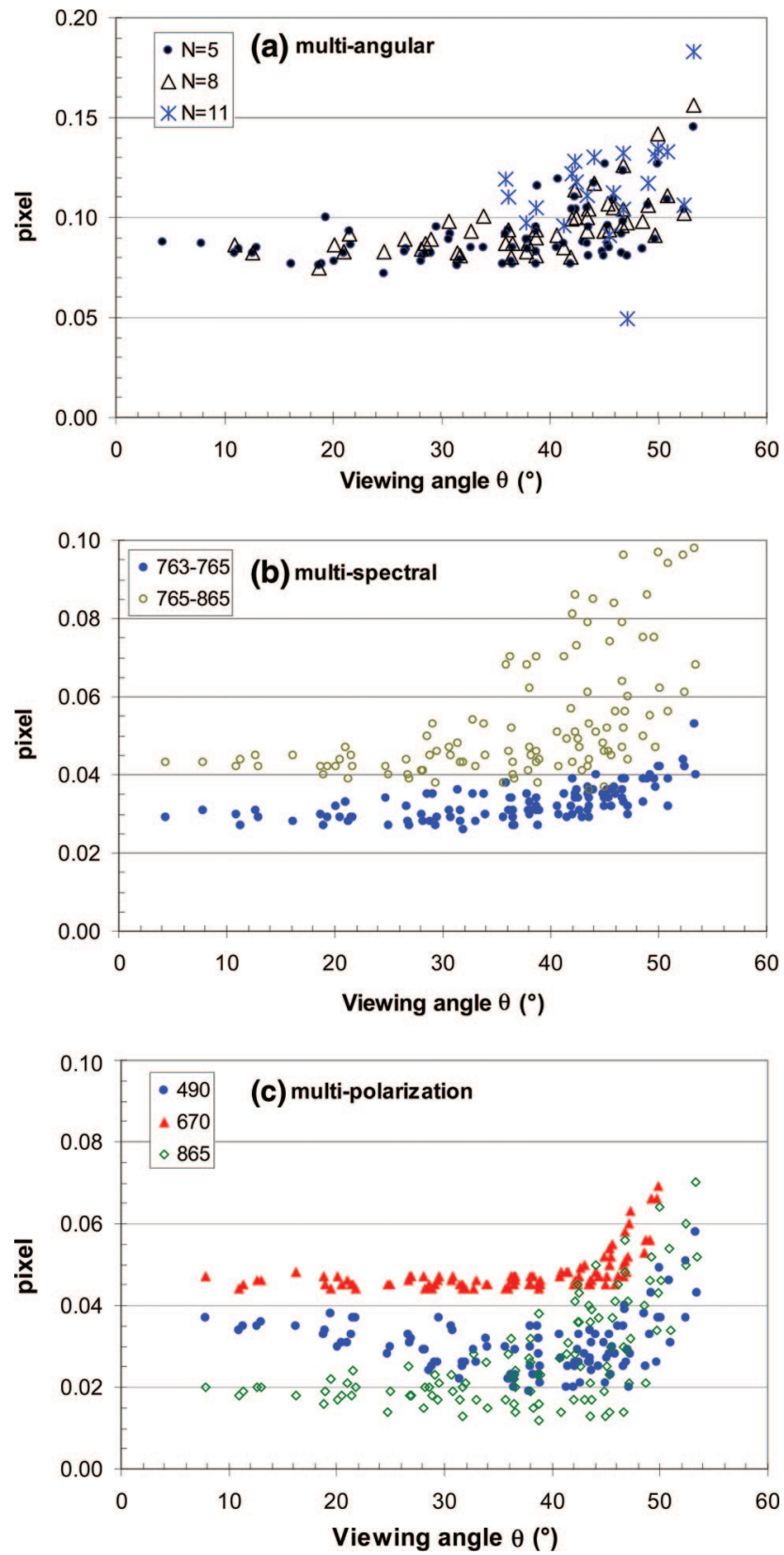

Fig. 14. (Color online) PARASOL geometric registration performances and behavior as a function of the viewing angle for multiangular [(a), top], multispectral [(b), middle], and multipolarization [(c), bottom] aspects. For each of the $11 \times 10$ areas dividing the CCD matrix, the mean of all available measurements is reported. Statistics are presented in Tables 7, 8, and 9. 
Table 7. Multipolarization Registration Performance for 490, 670, and 865 Polarized Triplets ${ }^{a}$

\begin{tabular}{|c|c|c|c|c|c|c|c|}
\hline \multirow[b]{2}{*}{ Measure (in pixel) } & \multirow[b]{2}{*}{ Npts } & \multicolumn{2}{|c|}{490} & \multicolumn{2}{|c|}{670} & \multicolumn{2}{|c|}{865} \\
\hline & & Mean & Stdev & Mean & Stdev & Mean & Stdev \\
\hline Range $\left[0^{\circ}-35^{\circ}\right]$ & 225959 & 0.0312 & 0.0042 & 0.0455 & 0.0010 & 0.0204 & 0.0031 \\
\hline Range $\left[35^{\circ}-45^{\circ}\right]$ & 223490 & 0.0263 & 0.0045 & 0.0458 & 0.0014 & 0.0263 & 0.0099 \\
\hline Range $\left[45^{\circ}-50^{\circ}\right]$ & 95421 & 0.0317 & 0.0070 & 0.0496 & 0.0042 & 0.0357 & 0.0130 \\
\hline Performance at $50^{\circ}$ & & 0.0347 & & 0.0530 & & 0.0431 & \\
\hline Specification & & 0.0500 & & 0.0500 & & 0.0500 & \\
\hline
\end{tabular}

${ }^{a}$ Mean, standard deviation and number of measurements are reported for 3 angular ranges, for the $50^{\circ}$ viewing angle, and confronted to the specification expressed in pixel.

up to 16. Each viewing angle is separated from the previous one by approximately 20 seconds and $9^{\circ}$ and is derivate from a different raw image [1]. On the level-1 data processing chain [5], the $N$ acquisitions are registered for each level-1 pixel with a given accuracy strongly dependent on residual biases on the geometric correction, i.e., on the calibration error. The multiangular registration performance is assessed by a correlation algorithm using images acquired for the band 765 and separated by $N$ viewing directions, $N$ varying from 1 to 16 . Table 9 reports registration performances for 7 values of $N$. The mean performances per area $(11 \times 10$ areas $)$ are illustrated in Fig. 14(a) where it is evidenced that the registration evaluation is increasing for large viewing angles: in fact, this evaluation is the real performance plus a correlation error that includes a sensible bidirectional effect of targets. Consequently, the real performance is within the estimation, and the specification is considered as met. The performance established during the commissioning phase in March 2005 has been verified after one year in orbit (Table 9) and the absence of temporal evolution proves that the instrumental alignment, i.e., the geometric calibration, is perfectly stable with time.

\section{Multispectral Registration}

The 9 spectral bands presented in Table 2 are not acquired simultaneously, but successively during one turn of the filter wheel in 20.096 seconds. The spectral information is registered on the geometric level-1 processing using the geometric model of the instrument (see Subsection 3.A) [5]. The multispectral registration performance is assessed by a correlation algorithm using images acquired for a couple of spectral bands. The estimated performance includes the real performance plus a correlation error depending on the observed target and on the considered wavelength. Figure 14(b) and Table 8, the estimation made for the couple of bands 763-765 is better than other couples by nearly a factor of 2 . This is explained by the fact that these 2 spectral bands observed a very similar spectral scene, only differing by an attenuation due to the dioxygen absorption. Figure 14(b) shows the 763-765 performances are considered as the more realistic evaluation for the real multispectral performance and are clearly under specification. The performance established during the commissioning phase has been verified after one year in orbit (Table 8), and the absence of temporal evolution proves that the instrumental geometric model is perfectly stable with time.

\section{Multipolarization Registration}

For a polarized triplet, 3 acquisitions are realized for a polarizer oriented at $-60^{\circ}(X-60), 0^{\circ}(X 0)$, and $+60^{\circ}$ (X60) from a given reference [1]. These 3 components are successively acquired in 1 second during when the observed ground target moves in the field-of-view due to the satellite progression, mainly along track, and the Earth rotation, mainly cross track. Onboard registration of the 3 components is ensured by prisms

Table 8. Multi-Spectral Registration Performance Estimated for 8 Couples of Neighbor Spectral Bands ${ }^{a}$

\begin{tabular}{|c|c|c|c|c|c|c|}
\hline \multirow[b]{2}{*}{ Bands } & \multicolumn{3}{|c|}{ March 2005} & \multicolumn{3}{|c|}{ March 2006} \\
\hline & Pixel & Npts & Stdev & Pixel & Npts & Stdev \\
\hline $443-490$ & 0.0778 & 40686 & 0.0158 & 0.0746 & 68847 & 0.0195 \\
\hline $490-565$ & 0.0895 & 33672 & 0.0147 & 0.0478 & 61191 & 0.0075 \\
\hline $565-670$ & 0.0924 & 31639 & 0.0134 & 0.0907 & 52080 & 0.0170 \\
\hline $670-763$ & 0.0607 & 34984 & 0.0122 & 0.0693 & 43635 & 0.0145 \\
\hline $763-765$ & 0.0342 & 50774 & 0.0046 & 0.0325 & 82513 & 0.0045 \\
\hline $765-865$ & 0.0520 & 47497 & 0.0155 & 0.0511 & 78461 & 0.0182 \\
\hline $865-910$ & 0.0627 & 44134 & 0.0099 & 0.0618 & 74917 & 0.0128 \\
\hline 910-1020 & 0.0794 & 38646 & 0.0198 & 0.0797 & 66373 & 0.0246 \\
\hline Specification & 0.1000 & & & 0.1000 & & \\
\hline
\end{tabular}

${ }^{a}$ Mean standard deviation, and number of measurements are reported for the commissioning phase in March 2005 and for the annual verification in March 2006. Values are confronted to the specification in pixel. 
Table 9. Multiangular Registration Performance Estimated for 7 Turn Numbers of the Filter Wheel ${ }^{a}$

\begin{tabular}{|c|c|c|c|c|c|c|}
\hline \multirow{2}{*}{$\begin{array}{l}\text { Number of } \\
\text { Directions }\end{array}$} & \multicolumn{3}{|c|}{ March 2005} & \multicolumn{3}{|c|}{ March 2006} \\
\hline & Pixel & Npts & Stdev & Pixel & Npts & Stdev \\
\hline 3 & 0.0836 & 60077 & 0.071 & 0.0851 & 31484 & 0.067 \\
\hline 5 & 0.0909 & 38999 & 0.074 & 0.0917 & 19374 & 0.067 \\
\hline 8 & 0.0981 & 20839 & 0.074 & 0.1074 & 9568 & 0.073 \\
\hline 11 & 0.1219 & 1932 & 0.082 & 0.1255 & 2468 & 0.081 \\
\hline 12 & 0.1339 & 1096 & 0.085 & 0.1318 & 1388 & 0.087 \\
\hline 13 & 0.1628 & 326 & 0.089 & 0.1529 & 419 & 0.098 \\
\hline 14 & 0.1370 & 3 & 0.056 & 0.2530 & 5 & 0.104 \\
\hline Specification & 0.1000 & & & 0.1000 & & \\
\hline
\end{tabular}

${ }^{a}$ Mean standard deviation, and number of measurements are reported for the commissioning phase in March 2005 and for the annual verification in March 2006. Values are confronted to the specification in pixel. Results for 14 viewing directions are considered as nonsignificant.

viewing forward (for $X-60)$ and backward (for X60) compensating the satellite progression, about $6 \mathrm{~km}$ per second, while the Earth rotation is compensated at first order by a yaw-steering of the satellite platform. The multipolarization registration performance of the polarized triplet, defined as the radius of the circle at the Earth surface containing the 3 components $X-60, X 0$, and $X 60$, is assessed by a correlation algorithm. Results are presented in Table 7 and illustrated in Fig. 14(c) as a function of the viewing angle. The performance for the band 670 is close to 0.05 and equivalent whatever the viewing angle (except for large angles near $50^{\circ}$ ), while the band 490 is more optimized on the $35-45^{\circ}$ range and the band 865 more optimized for near-nadir viewing angle in the range $0-35^{\circ}$. Performances estimated during the commissioning phase were reevaluated after one year in orbit, and no temporal evolution has been detected.

\section{Absolute Location Accuracy}

The absolute location of level-1 data product was estimated using ground control manual pointing and using as reference VEGETATION/SPOT5 products for which the absolute location accuracy is within $150 \mathrm{~m}$ compared to the $6 \mathrm{~km}$ of the PARASOL resolution [34]. A few Vegetation products were ordered at the CTIV with the same specific reprojection than PARASOL products (equal area sinusoidal projection). Images were selected over France, Australia, Arabia, and Canada in mid 2005. Table 10 shows

\begin{tabular}{lcrrrr} 
Table 10. & $\begin{array}{c}\text { Absolute Location Accuracy Estimation Using VEgetation } \\
\text { SPOT5 as Reference }\end{array}$ \\
& Npts & Mean & Stdev & Min & \multicolumn{1}{c}{ Max } \\
\hline$X$ (in pixel) & 96 & $-0,193$ & 0,242 & 0,683 & $-0,610$ \\
$Y$ (in pixel) & 96 & 0,097 & 0,256 & 0,718 & $-0,413$ \\
Norm (in pixel) & 96 & 0,366 & 0,191 & 0,832 & 0,040 \\
Norm (km) & 96 & 2,26 & 1,18 & 5,14 & 0,24 \\
Specification (km) & & 4,00 & & & \\
Objective (km) & & 2,00 & & & \\
\hline
\end{tabular}

${ }^{a}$ Mean, standard deviation, minimum and maximum values, and number of measurements are reported for $X$ axis (along track), $Y$ axis (cross track), and norm of the deviation in pixel and in $\mathrm{km}$, and finally confronted to specifications. statistics for nearly one hundred points of measurements and a very good performance close to $2 \mathrm{~km}$. Specification is met and goal performance is nearly satisfied.

\section{Conclusion}

In-flight characterization and validation of preflight estimations were presented for both radiometric and geometric aspects. PARASOL in-flight performances were evaluated during the commissioning phase in spring 2005 regarding to the mission specifications, and concluding to adequate level-1 product quality for scientific exploitation. The only identified limitation was a very poor quality of the band 443 due to an unresolved stray-light problems, and it is strongly recommended not to use measurements from this spectral band. After one year in orbit, performances were re-evaluated: All radiometric and geometric performances were confirmed, even if a light radiometric temporal decrease was detected, modeled, and corrected conducting to a rereprocessing of the entire level-1 data archive ended in November 2006 [35]. The new Version 3 PARASOL level-1 archive is available at CPP (Centre de Production POLDER) at http:// polder.cnes.fr/.

\section{Appendix A: Acronyms}

ADEOS Advanced Earth Observing SatelCALIPSO Cloud-Aerosol Lidar and Infrared CCC Centre de Commande Contrôle CCD Charge coupled device

CERES Clouds and Earth's radiant energy CLOUDSAT $\quad$ CLOUD Satellite CNES Centre National d'Etudes Spatiales CPP Centre de Production POLDER

CTIV Centre de Traitement des Images ENVISAT Environmental Satellite LIT Long integration time MERIS Medium Resolution Imaging Spectrometer 
MODIS

NedI

NedIp

NIR

PARASOL

POLDER

SeaWiFs

SIT

SQI

SPOT

SRI

TOA

We are very grateful to all those who have contributed to the elaboration of this large collection of concluding results mainly at the PARASOL project, CNES/CCC (Centre de Commande Contrôle), CNES/ CPP (Centre de Production POLDER) and CNES/SQI (Système Qualité Image PARASOL): M. Bach, P. Lier, F. Serene, P. Henry, C. Dabin, R. Reich, F. BaillyPoirot, E. Orsal, E. Tolsan, E. Espié, F. Meunier, J. Rollin, R. Bach, S. Leguevaques, D. Toustou, and scientists from Laboratoire d'Optique Atmosphérique (LOA, Lille, France) and Laboratoire des Sciences du Climat et de I'Environnement (LSCE, Saclay, France) for their helpful discussions.

\section{References}

1. P. Y. Deschamps, F.-M. Bréon, M. Leroy, A. Podaire, A. Bricaud, J.-C. Buriez, and G. Sèze, "The POLDER mission: instrument characteristics and scientific objectives," IEEE Trans. Geosci. Remote Sens. 32, 3598-3615 (1994).

2. M. Thoby, "Myriad: CNES Micro-Satellite Program," presented at the 15th Annual AIAA/USU Conference on Small Satellites, Logan, Utah, 13-16 August 2001, paper SCO1-I-8.

3. B. Fougnie, G. Bracco, B. Lafrance, C. Ruffel, O. Hagolle, and C. Tinel, "In-flight performances of PARASOL inside the aquatrain atmospheric observatory," presented at the AGU Fall Meeting, San Francisco, California, 5-9 December 2005, paper A33C-915.

4. O. Hagolle, P. Goloub, P.-Y. Deschamps, H. Cosnefroy, X. Briottet, T. Bailleul, J. M. Nicolas, F. Parol, B. Lafrance, and M. Herman, "Results of POLDER in-flight calibration," IEEE Trans. Geosci. Remote Sens. 37, 1550-1566 (1999).

5. O. Hagolle, A. Guerry, L. Cunin, B. Millet, J. Perbos, J. M. Laherrère, T. Bret-Dibat, and L. Poutier, "POLDER level 1 processing algorithms," in Algorithms for Multispectral and Hyperspectral Imagery II, Proc. SPIE 2758, pp. 308-319 (1996).

6. T. Bret-Dibat, Y. Andre, and J. M. Laherrère, "Preflight calibration of the POLDER instrument," Remote Sensing and Reconstruction for three Dimensional Objects and Scenes, Proc. SPIE 2572, doi:10.1117/12.221357 (1995).

7. B. Lafrance, C. Ruffel, and B. Fougnie, "PARASOL pre-flight radiometric calibration-synthesis report," CNES Tech. Memo. PAR-NT-S7-6423-CNS (Centre National d'Etudes Spatiales, Toulouse, France, 2005).
8. B. Fougnie, P. Henry, F. Cabot, A. Meygret, and M.-C. Laubies, "VEGETATION: in-flight multi-angular calibration," in Earth Observing Systems V, Proc. SPIE 4135, 331-338 (2000).

9. B. Fougnie, O. Hagolle, and F. Cabot, "In-flight measurement and correction of non-linearity of the POLDER-1's sensitivity," presented at the 8th Symposium of the International Society for Photogrammetry and Remote Sensing, Aussois, France, 8-12 January 2001.

10. J. M. Laherrère, L. Poutier, T. Bret-Dibat, O. Hagolle, C. Baqué, P. Moyer, and E. Verges, "POLDER on-ground stray light analysis, calibration and correction," presented at $E u$ ropto Conference on Sensors, Systems and Next Generation Satellites III, London, UK, 22-26 September 1997.

11. R. E. Eplee, Sean W. Bailey D. Wayne D. Robinson D. K. Clark, P. J. Werdell, M. Wang, R. A. Barnes, and C. R. McClain, "Calibration of SeaWiFS. II. Vicarious Techniques," Appl. Opt. 40, 6701-6718 (2001).

12. O. Hagolle and F. Cabot, "Calibration of MERIS using natural targets," presented at the Second MERIS and AATSR Calibration and Geophysical Validation Workshop, Frascati, Italy, 20-24 March 2006.

13. B. Fougnie, P. Y. Deschamps, and R. Frouin, "Vicarious Calibration of the POLDER ocean color spectral bands using in-situ measurements," IEEE Trans. Geosci. Remote Sens. 37, 1567-1574 (1999).

14. B. Fougnie, B. P. Henry, A. Morel, D. Antoine, and F. Montagner, "Identification and characterization of stable homogeneous oceanic zones: climatology and impact on in-flight calibration of space sensor over Rayleigh scattering," presented at Ocean Optics XVI, Santa Fe, New Mexico, 18-22 November 2002.

15. E. Shettle and R. Fenn, "Models for the aerosols of the lower atmosphere and the effects of humidity variations on their optical properties," AGFL-TR-79-0214, U.S. Air Force Geophys. Lab. (Hanscom Air Force Base, 1979), p. 94.

16. E. Vermote, R. Santer, P.-Y. Deschamps, and M. Herman, "In-flight calibration of large field of view sensors at shorter wavelengths using Rayleigh scattering," Int. J. Remote Sens. 13, 3409-3429 (1992).

17. C. Cox and W. Munk, "Measurements of the roughness of the sea surface from photographs of the sun's glitter," J. Opt. Soc. Am. 44, 838-850 (1954)

18. O. Hagolle, J. M. Nicolas, B. Fougnie, F. Cabot, and P. Henry, "Absolute calibration of VEGETATION derived from an interband method based on sunglint over ocean sites," IEEE Trans. Geosci. Remote Sens. 42, 1-10 (2004).

19. B. Toubbé, T. Bailleul, J. L. Deuzé, P. Goloub, O. Hagolle, and M. Herman, "In-flight calibration of the POLDER polarized channels using the sun's glitter," IEEE Trans. Geosci. Remote Sens. 37, 513-525 (1999).

20. C. Vanbauce, J. C. Buriez, F. Parol, B. Bonnel, G. Sèze, and P. Couvert, "Apparent pressure derived from ADEOS-POLDER observations in the oxygen A-band over ocean," Geophys. Res. Lett. 25, 3159-3162 (1998).

21. B. Lafrance, O. Hagolle, B. Bonnel, Y. Fouquart, and G. Brogniez, "Interband calibration over clouds for POLDER space sensor," IEEE Trans. Geosci. Remote Sens. 40, 131-142 (2002).

22. H. Cosnefroy, M. Leroy, and X. Briottet, "Selection and characterization of Saharian and Arabian desert sites for the calibration of optical satellite sensors," Remote Sens. Environ. 58, 101-114 (1996).

23. F. Cabot, O. Hagolle, C. Ruffel, and P. Henry, "Remote sensing data repository for in-flight calibration of optical sensors over terrestrial targets," in Earth Observing Systems IV, Proc. SPIE 3750, 514-523 (1999).

24. B. Fougnie, F. Cabot, O. Hagolle, and P. Henry, "CNES con- 
tribution to ocean color calibration: cross-calibration over desert sites," SIMBIOS Project 2001 Annual Report, NASA/ TM-2002-210005 (NASA, 2002), pp. 159-163.

25. H. Rahman and G. Dedieu, "SMAC: a simplified method for the atmospheric correction of satellite measurements in the solar spectrum," Int. J. Remote Sens. 15, 123-143 (1994).

26. R. A. Barnes and F. Zalewski, "Reflectance-based calibration of SeaWiFS: I. Calibration coefficients," Appl. Opt. 42, 16291647 (2003).

27. L. Bourg and S. Delwart, "MERIS instrument calibration," presented at the Second MERIS and AATSR Calibration and Geophysical Validation Workshop, Frascati, Italy, 20-24 March 2006.

28. X. Xiong and W. Barnes, "An overview of MODIS radiometric calibration and characterization," Adv. Atmos. Sci. 23, 69-79 (2006).

29. R. A. Barnes, R. E. Eplee, G. M. Schmidt, F. S. Platt, and C. R. McClain, "Calibration of SeaWiFS. I. Direct techniques," Appl. Opt. 40, 6682-6700 (2001).

30. D. Six, M. Fily, S. Alvain, P. Henry, and J. P. Benoist, "Surface characterization of the Dome Concordia area (Antarctica) as a potential satellite calibration site, using SPOT-4 VEGETATION instrument," Remote Sens. Environ. 89, 83-94 (2004).

31. IOCCG, "In-flight calibration of ocean Color Sensor," Reports of the International Ocean-Color Coordinating Group, R. Frouin, ed. (Dartmouth, Canada, to be published).

32. J. C. Buriez, C. Vanbauce, F. Parol, P. Goloub, M. Herman, B. Bonnel, Y. Fouquart, P. Couvert, and G. Sèze, "Cloud detection and derivation of cloud properties from POLDER," Int. J. Remote Sens. 18, 2785-2813 (1997).

33. N. A. J. Schutgens, L. G. Tilstra, P. Stamnes, and F. M. Bréon, "On the relationship between Stokes parameters Q and U of atmospheric ultraviolet/visible/near-infrared radiation," J. Geophys. Res. 109, D09205, doi:10.1029/2003JD004081 (2004).

34. S. Sylvander, I. Albert-Grousset, and P. Henry, "Geometric performances of the VEGETATION products," IEEE International Geosciences And Remote Sensing Symposium (IEEE, 2003), Vol. 1, pp. 573-575.

35. http://polder.cnes.fr/en/news/index.htm. 